\title{
Test-Bench for the Characterization of Flexion Sensors Used in Biomechanics
}

\author{
Monica Tiboni ${ }^{1, *(\mathbb{D}}$, Azzurra Filippini $^{2}$, Cinzia Amici ${ }^{1}$ (D) and David Vetturi ${ }^{1}$ (D) \\ 1 Department of Mechanical and Industrial Engineering, University of Brescia, Via Branze, 38, \\ 25123 Brescia, Italy; cinzia.amici@unibs.it (C.A.); david.vetturi@unibs.it (D.V.) \\ 2 Ocrim s.p.a., Via Massarotti, 76, 26100 Cremona, Italy; a.filippini@ocrim.com \\ * Correspondence: monica.tiboni@unibs.it
}

check for updates

Citation: Tiboni, M.; Filippini, A.; Amici, C.; Vetturi, D. Test-Bench for the Characterization of Flexion Sensors Used in Biomechanics. Electronics 2021, 10, 2994. https:// doi.org/10.3390/electronics10232994

Academic Editors: Flavio Canavero and Enzo Pasquale Scilingo

Received: 26 October 2021

Accepted: 29 November 2021

Published: 1 December 2021

Publisher's Note: MDPI stays neutral with regard to jurisdictional claims in published maps and institutional affiliations.

Copyright: (c) 2021 by the authors. Licensee MDPI, Basel, Switzerland. This article is an open access article distributed under the terms and conditions of the Creative Commons Attribution (CC BY) license (https:// creativecommons.org/licenses/by/ $4.0 /)$.

\begin{abstract}
The design, prototyping and validation of an innovative test bench for the characterization and the hysteresis measurement of flexion sensors are presented in this paper. The device, especially designed to test sensors employed in the biomedical field, can be effectively used to characterize also sensors intended for other applications, such as wearable devices. Flexion sensors are widely adopted in devices for biomedical purposes and in this context are commonly used in two main ways: to measure movements (i) with fixed radius of curvature and (ii) with variable radius of curvature. The test bench has been conceived and designed with reference to both of these needs of use. The technological choices have been oriented towards simplicity of manufacture and assembly, configuration flexibility and low cost of realization. For this purpose, 3D printing technology was chosen for most of the structural components of the device. To verify the test bench performances, a test campaign was carried out on five commercial bending sensors. To characterize each sensor, the acquired measurements were analysed by assessing repeatability and linearity of the sensors and hysteresis of the system sensor/test bench. A statistical analysis was performed to study the positioning repeatability and the hysteresis of the device. The results demonstrate good repeatability and low hysteresis.
\end{abstract}

Keywords: flexion sensors; characterization; test-bench; biomechanics; wearable devices

\section{Introduction}

The measurement of flexion or extension of specific elements in a system is a requirement that can arise in many application fields. Flex sensors, known also as flexion or bending sensors, perform this task. Flex sensor applications can be classified into two principal categories [1]: measurement of static and dynamic human body postures and movements detection in artificial devices.

Many publications concern the employment of flexion sensors for biometric measurements, especially as electronic goniometers. These research works differ in the part of the body whose movement is detected and in the application of the measure. There are use cases for the upper limbs [2], lower limbs [3,4] and for head and torso [5]. The purposes range from medical, to the recognition of gestures, to the creation of human/machine interfaces. Some works discuss the use of flex sensors for the evaluation of the range of motion (ROM), for example for clinical monitoring in rehabilitation [6-8] or for monitoring body postures [9,10]. Many publications concern the development or application of instrumented gloves with flexion sensors to control exoskeletons for different purposes, such as: hand rehabilitation [11], and more generally, control of robotic devices [12-14], translation of sign language [15], recognition of hand gestures [16,17], development of an advanced human/computer interface [18], tele-operation [13] and prosthetic devices control [19,20].

Artificial systems with flex sensors can be found in various fields. Flexion sensors are widely used in wearable devices [21,22], both with health monitoring function and for measuring joint ranges. An application in the automotive industry concerns the creation of 
safety systems against the damages that can be caused by an airbag on children or adults with a body mass index below the average [23]. Flex sensors are effectively used in robotic devices: in [24] the action of a 3D printed robotic forearm is controlled by a glove with flex sensors, in [25] flexion sensors are used in a Master-Slave robotic hand system and in [26] a combination of flex sensor and electromyography is used for hybrid robotic control. Within the emerging technique of soft actuators, various works involving flexion sensors can be found $[27,28]$. Very singular applications are a soft robot inspired by frogs and with integrated bending sensors [29] or a wireless air flow transmitter using flex sensor [30].

Resistive Flex Sensors (RFSs) are the most common version of flexion sensors, and their functioning principle is the electrical resistance variation of a conductive material with the bending. They are formed by a resistive element placed on or inside a thin flexible substrate that is able to tolerate flexion, vibration, thermal shock and stretching [31]. The adopted conductive material and the way it is layered determine the basic electrical properties of the sensor. In this way, the sensor resistance is highly susceptible to any defects along the conductive surface.

Different solutions are available, and others are the object of research and experimentation for the manufacturing of RFSs. The conductive component more frequently is based on carbon elements, which can be in the form of ink, particles, powder, nanoparticles, nanotubes [1] or different forms of carbon as graphite or graphene [32,33]. Alternatives are transition metals (i.e., silver, platinum, copper, nichel) [34], conductive polymers [35] or composites [36].

Regardless of the field of application and of the constructive technology, the characterization concerns a very important aspect for the correct use of a bending sensor as well as for all sensors.

The characterization of the sensors involves finding their characteristic curve, that is the relationship between the physical quantity at the input and the one at the output. This function is calculated by measuring the sensor output quantity for known values of the input and in static conditions, i.e., the sensor must be in normal operating conditions (temperature, humidity and pressure), the variation of the input has to be slow and external stresses (shocks, vibrations, accelerations) are not allowed.

The used method of characterization of the sensors varies according to the joint to which they are applied. In fact, the flexion and deformation sensors that are used as electronic goniometers can adapt to any type of joint, but they must be characterized differently depending on the size of the joint. This is because, in the case of small dimensions of the joint (fingers [37], elbow [38]), the sensor is bent only in the central area and its resistivity increases only in that area; furthermore, the radius of curvature is fixed. Instead, in the case of large dimensions (knee [3,39,40], trunk posture [41], neck movement: radius of curvature wider than the length of the sensor) the sensor bends evenly with variable radius of curvature and the resistivity increases along its entire length. In the first case, the sensor can be characterized on a mobile hinge with a constant radius of curvature, using the range of motion of the considered joint (for example, $90^{\circ}$ for the finger). In [42] Saggio and others present a characterization device in which one side of the sensor is locked in a fixed gripper, placed on a rotating platform driven by a stepper motor, while the other side is placed in a sliding clamp to avoid stretching the sensor. The disadvantage of this method, however, is that the characteristic curve of the sensor, and therefore its behaviour, depends on the radius of the hinge. Therefore, since these sensors can be applied to different sized body joints, the characterization procedure should be repeated for a wide range of hinge diameter values [16].

To characterize sensors to be used with wide radii of curvature, it is necessary to take into account that the sensor bends with a uniform radius of curvature that varies over time, so the resistance also increases uniformly over the entire resistive layer. In [43] to carry out this type of characterization, authors use a screw shaft, driven by a step motor, which moves a carriage in push or pull. The sensor is fixed on a flexible support that is locked on the sliding carriage on one side and on a fixed support on the other. When the step motor 
is activated, the elastic support bends causing uniform flexion of the sensor, with a radius of curvature depending on the position of the motor. This characterization method is valid for all sensors of the same technology regardless of their size, provided that their length is less than or equal to the radius of curvature.

A test bench consisting of a folding device with three different diameters to flex the sensors and an encoder for measuring the movements was developed and used to characterize sensors by varying the bending angles [44].

However, the prototypal test benches described in literature allow for the characterization of the sensors only in one modality; there are no test benches that can be used for characterization with both fixed and variable radius of curvature or that can be used for both bending sensors and strain sensors.

In this paper a new test bench for the characterization of flex sensors is presented. The innovative aspects of this device are that it is employable both for fixed and variable curvature radii applications, it can be used for flexion sensors but also for strain sensors, it is a low cost-device for which a significant part of the components is made by $3 \mathrm{D}$ additive manufacturing and it is easy to use.

\section{Materials and Methods}

\subsection{Mechanical Structure of the Test Bench}

In the design phases of the test bench, the following technical specifications were defined to be respected:

- Adaptability of the device to both cases: sensors used with fixed and variable radius of curvature;

- High measurement repeatability;

- Simplicity of use;

- Ease of reproduction;

- Low cost of construction;

- Possibility of using the device for both flexion and strain sensors.

To comply with these specifications, the following technical choices have been made:

- Linear movement of the element connected to the component on which the sensor is arranged to generate the bending, using a linear motor with high repeatability;

- Realization of two different types of sensor support element to obtain the two different bending modalities required by the two main types of applications as a function of the radius of curvature;

- Design of a significant number of components suitable for 3D printing with additive manufacturing;

- Design and implementation of the hardware infrastructure for the acquisition of measurements with a low-cost and widely used device of the Arduino family;

- Development of the software for the acquisition of the measurements and the subsequent storage and reprocessing in an advanced environment with an easy-to-use graphic interface to make the characterization operations of a significant number of sensors as efficient as possible.

The 3D representation of the mechanical structure of the test bench prototype made by adopting the aforementioned choices is visible in Figures 1 and 2. Figure 1 shows the configuration for the characterization of sensors employed with variable radius of curvature, while Figure 2 shows the configuration for applications with constant radius of curvature. 


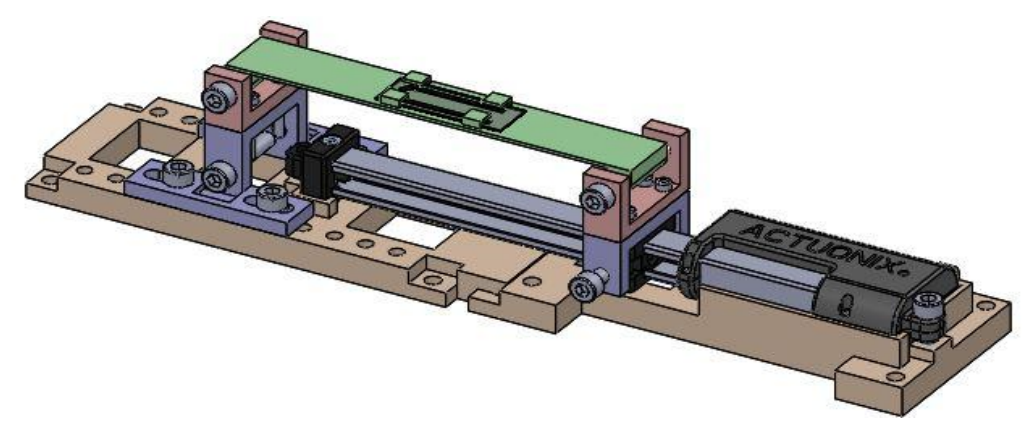

Figure 1. 3D representation of the mechanical structure of the test bench for the characterization of sensors for applications with variable radius of curvature.

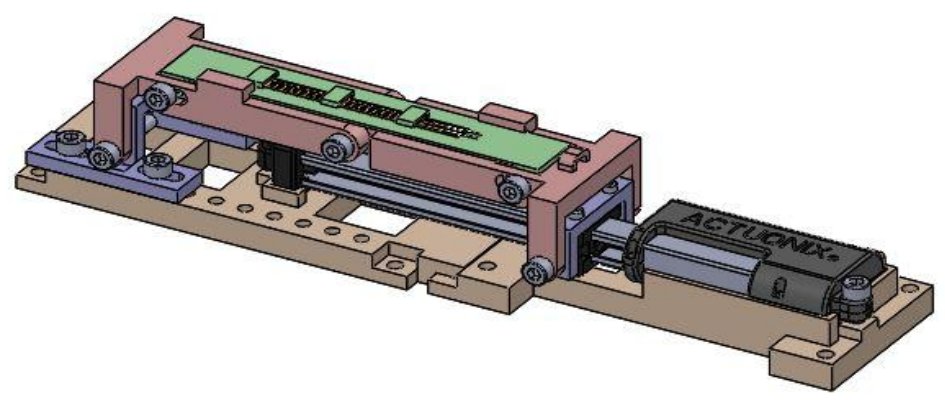

Figure 2. 3D representation of the mechanical structure of the test bench for the characterization of sensors for applications with constant radius of curvature.

The structural and complementary parts of the test bench designed have been printed with different materials: TPU for flexible components and PETG and ABS for rigid parts. The base and the actuation part of the system is common to the two different cases of employment. The used actuator is a $12 \mathrm{~V}$, T16-P Miniature Track by Actuonix, with conversion of the motion from rotary to linear by means of a ball screw. It has a stroke of $100 \mathrm{~mm}$, gear ratio of $22: 1$, maximum speed of $46 \mathrm{~mm} / \mathrm{s}$, maximum force of $50 \mathrm{~N}$ and is rated force of $25 \mathrm{~N}$. The motor inside the linear actuator is a rotary direct current motor and a planetary gearbox transforms the rotary motion into linear motion. The actuator is mounted on a base, made of ABS, that allows it to be centred and fixed, thus preventing the actuator from moving during the characterization. For the characterization of sensors used with variable radius of curvature, the sensor is positioned on a flexible lamina, connected at one end to a mobile support. This support can slide between two guides and, after the lamina positioning, it is fixed to the base by bolts. The other side of the lamina is fixed to a support screwed onto the motor slider. This mechanism is useful to characterize the sensor starting from the initial position of the actuator and it also allows larger foils and sensors to be mounted on the test bench. The lamina is made in TPU; this solution is adequate because, in the post-production phase, the flexibility of the TPU components was tested and found to be flexible and bendable, perfectly meeting the needs of the application. The connection between lamina and supports is obtained through the coupling of cylindrical through holes at the ends of the foil with screws on the support to obtain the bending of the sheet as naturally as possible. On the lamina upper surface, three small slots have been obtained, inside which the flexion sensor can slide to be positioned (Figure 3) and, therefore, fixed to the foil, so that it can be characterized. With this fixing, the sensor is resting on the foil and is not rigidly connected to it; therefore, it is free to move along the bending direction but is constrained not to move in the other directions, so that only its deformation is not limited. Different shapes and positions of the slots may be easily obtained with 3D printing to adapt the lamina to different sensors shapes (as shown in Figure 3). The lamina material and thickness have been appropriately chosen, so as to 
obtain a bending as independent as possible from the physical characteristics of the sensor, therefore allowing a curvature that does not vary with the characterized sensor.

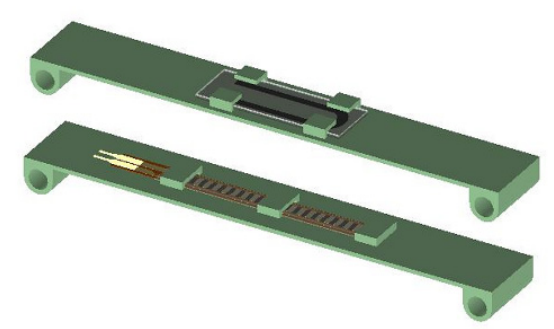

Figure 3. 3D representation of the lamina for the positioning of different sensors on the test bench.

The support fixed to the base has slots for fixing and a series of holes have been drilled in the base, so that the support can be fixed at the correct distance from the actuator by centring the holes with the slots (Figure 4).

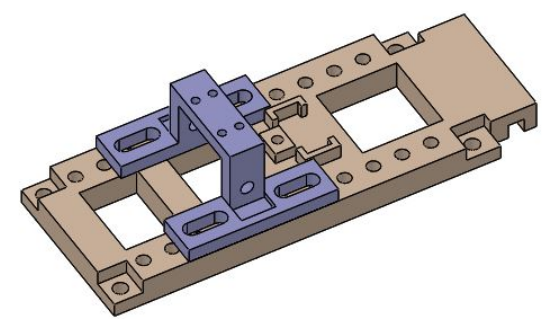

Figure 4. Guide-side support.

For the characterization of sensors used with fixed radius of curvature, the mounting of the lamina on the test bench must be different compared to the one described in the previous case. It is positioned on a V structure (Figure 5) formed by two rigid elements (pink in Figure 5) obtained in PTG and respectively connected to the two supports (fixed and mobile), so to create an angle when the actuator is operated. The lamina follows the angle formed by the two elements. The characteristic curve of a sensor depends on the radius of curvature with which it is bent. For this reason, ideally, a different characterization should be performed for each radius of curvature of the studied joint. For the developed prototype, the characterization of sensors used in the detection of the flexion of the fingers of the hand was considered, which, as highlighted in the introduction, represents a very widespread application of these sensors. In the case of the proximal interphalangeal joint, the angle depends on the size of the hand and the individual fingers. Since the range of variation of the radius of curvature of this joint is extremely small, good results can also be achieved by choosing an intermediate value for characterization. The radius of curvature of the proximal interphalangeal joint is determined by the head diameter of the proximal phalanx. According to the anthropometric tables in literature [45], taking into account all fingers of both hands and considering both female and male samples, this diameter ranges between $6 \mathrm{~mm}$ and $11 \mathrm{~mm}$, while its average value is $8.67 \mathrm{~mm}$. Considering these values, a cylindrical attachment was designed to connect the two rigid foils, which are joined by a bolt system, and a bending diameter of $12 \mathrm{~mm}$ was chosen. This value was obtained by rounding up the average diameter of the head of the phalanx to $9 \mathrm{~mm}$ and by adding $3 \mathrm{~mm}$ to take into account the thickness of tendons, ligaments and skin overlying the articulation. The obtained structure makes it possible to have a variable flexion angle and a fixed radius of curvature, allowing the sensor used to be characterized correctly. The connection between the elastic lamina and the V structure is obtained through slots; in this way, when the lamina is bent, its fixings can slide, so that the lamina is not put into traction and does not block the entire mechanism. The rigid element on the actuator side has been 
shaped so that it can be positioned above the carriage and its support, and a slot has been added in the upper part for the passage of the sensor cables (Figure 6).

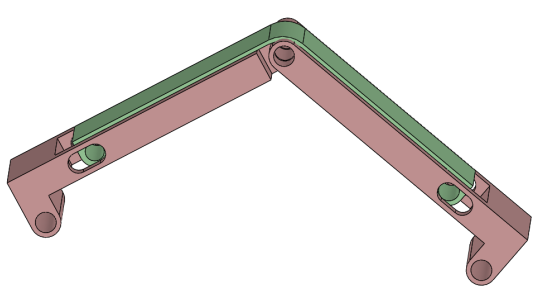

Figure 5. V structure for the elastic lamina support in the case of characterization of sensors in applications with fixed radius of curvature.

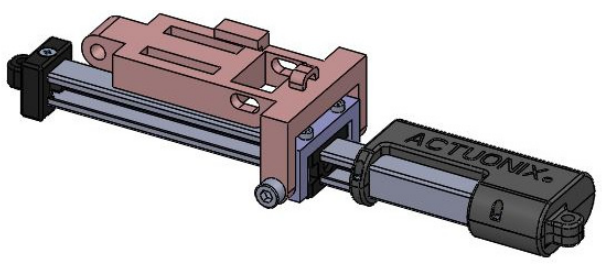

Figure 6. Actuator side element (pink) of the $\mathrm{V}$ structure for the elastic lamina support in the case of characterization of sensors used with fixed radius of curvature.

Figures 7 and 8 show the test bench prototype adapted to the two cases: characterization of sensors used with variable and fixed radius of curvature respectively. It allows the characterization of sensors used in the case of both large joints (by using only the configuration of Figure 7) and small joints (with the configuration of Figure 8). In addition, by regulating the position of the fixed support, it is possible to characterize even larger sensors by simply changing the rigid and elastic foils dimensions. In fact, each component is designed to be easily assembled and disassembled, allowing a quick setup even if it needs to be modified frequently. The designed test bench can also be used for the characterization of strain sensors, adopting the configuration of Figure 7, by replacing the flexible lamina with an extendable one and using the actuator in traction instead of thrust.

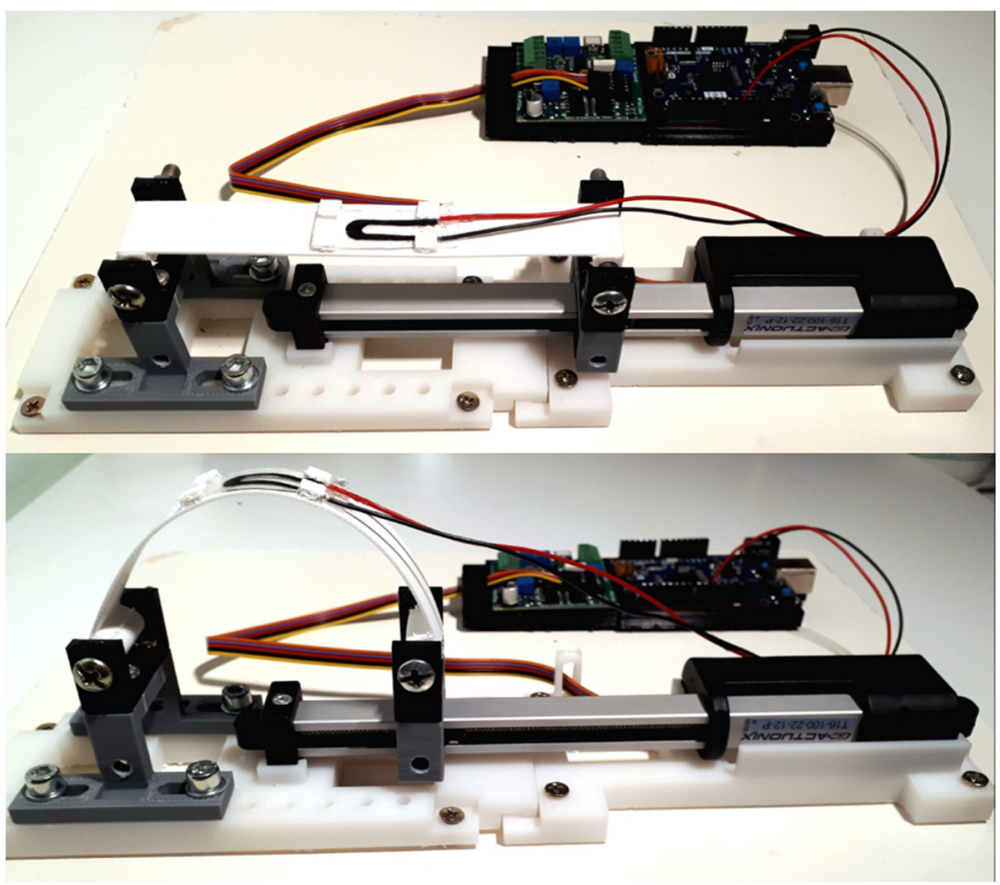

Figure 7. Test bench for sensors used for variable radius of curvature. 


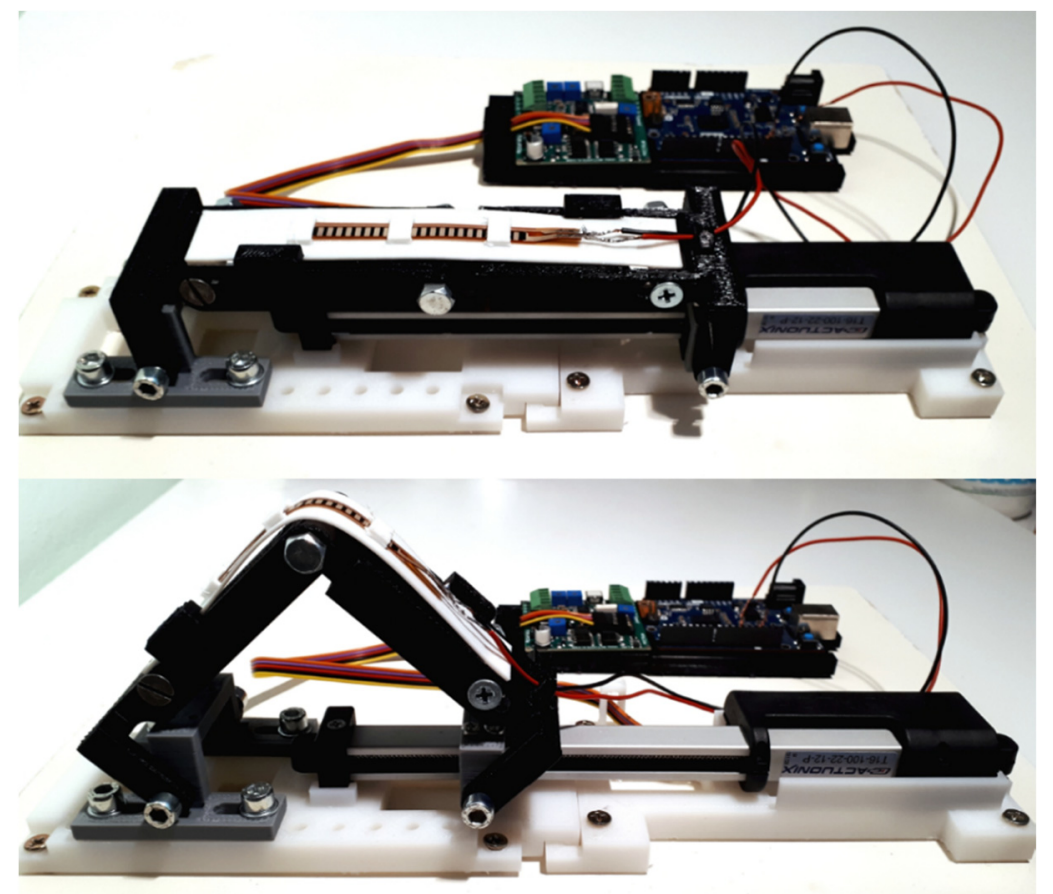

Figure 8. Test bench for sensors used for fixed radius of curvature.

\subsection{Hardware Structure of the Test Bench}

The test bench is formed by four main parts: the mechanical structure (described in paragraph 1), the flexion or deformation sensor to be characterized, a sensor electronic circuit and the electronic devices necessary for the acquisition of sensor signals and for the external interface for data collection and subsequent data processing. A schematic representation with block diagram of the overall architecture of the test bench is shown in Figure 9 .

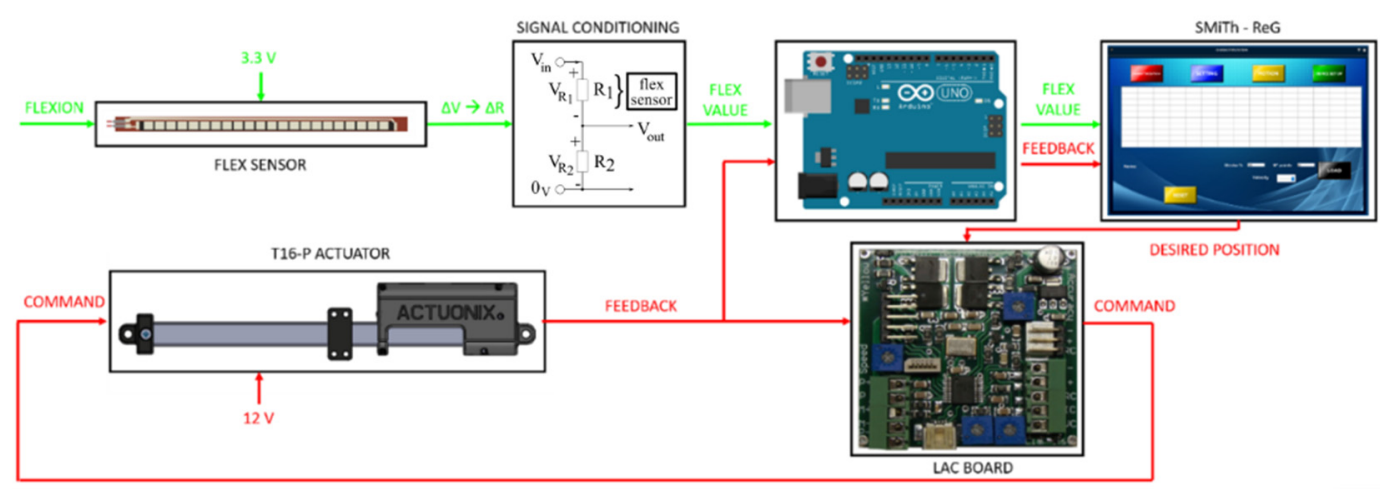

Figure 9. Block diagram of the test bench hardware structure.

The considered flexion sensors are of the resistive type, i.e., they have an internal resistance that varies when they are bent (flexion sensor) or deformed (strain sensor). In order to read this internal resistance variation, it is necessary to use a circuit in which the sensor acts as a voltage divider (Figures 10 and 11). Figure 10 shows the basic flex sensor circuit, as reported on the sensor datasheets. The impedance buffer is a single sided operational amplifier, used with these sensors because the low bias current of the operational amplifier (as LM358 or LM324) reduces error due to source impedance of the flex sensor as voltage divider. As further stated in the datasheet, it is possible to adopt a 
simpler circuit, skipping the operational amplifier; this is the solution adopted (Figure 11). The circuit has been built using a known resistance and an input voltage of $3.3 \mathrm{~V}$.

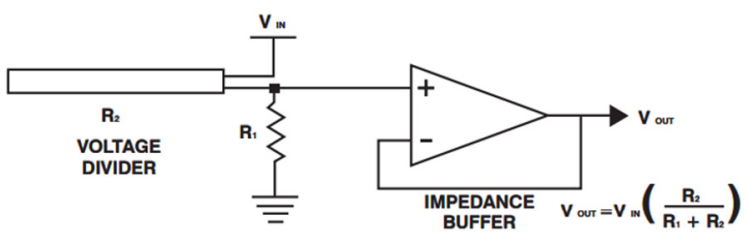

Figure 10. Sensor circuit.
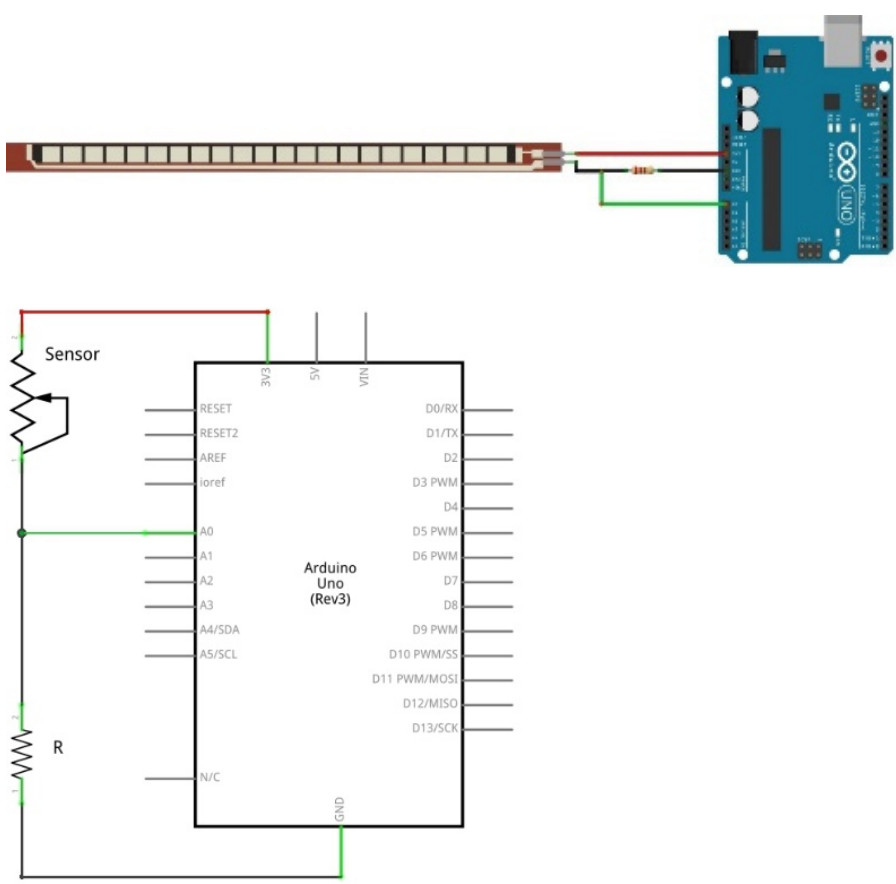

Figure 11. Arduino-sensor circuit for the test bench.

The linear actuator chosen for the test bench has no internal control, but it is equipped with a position sensor which provides an analog position feedback signal that can be fed into an external controller. It is furthermore equipped with a stand-alone, closed-loop control board (Linear Actuator Control Board, LAC) that can be used to control the actuator and which allows speed, sensitivity and stroke adjustments. The position transducer is a linear potentiometer. The effective resolution after ADC is $0.09 \mathrm{~mm}$. The feedback linearity is less than $2 \%$ and the declared repeatability is $\pm 0.4 \mathrm{~mm}$. The sensitivity or accuracy of the actuator control algorithm can be set by adjusting the "Accuracy" trim potentiometer on the LAC board. A final value of $0.033 \mathrm{~V} / \mathrm{mm}$ was settled. The LAC board can be used as a controller in different configurations. The most suitable in this application is the RC Servo Interface Mode (Figure 12): it is a standard hobby-type remote-control digital servo interface compatible with servo motors. 


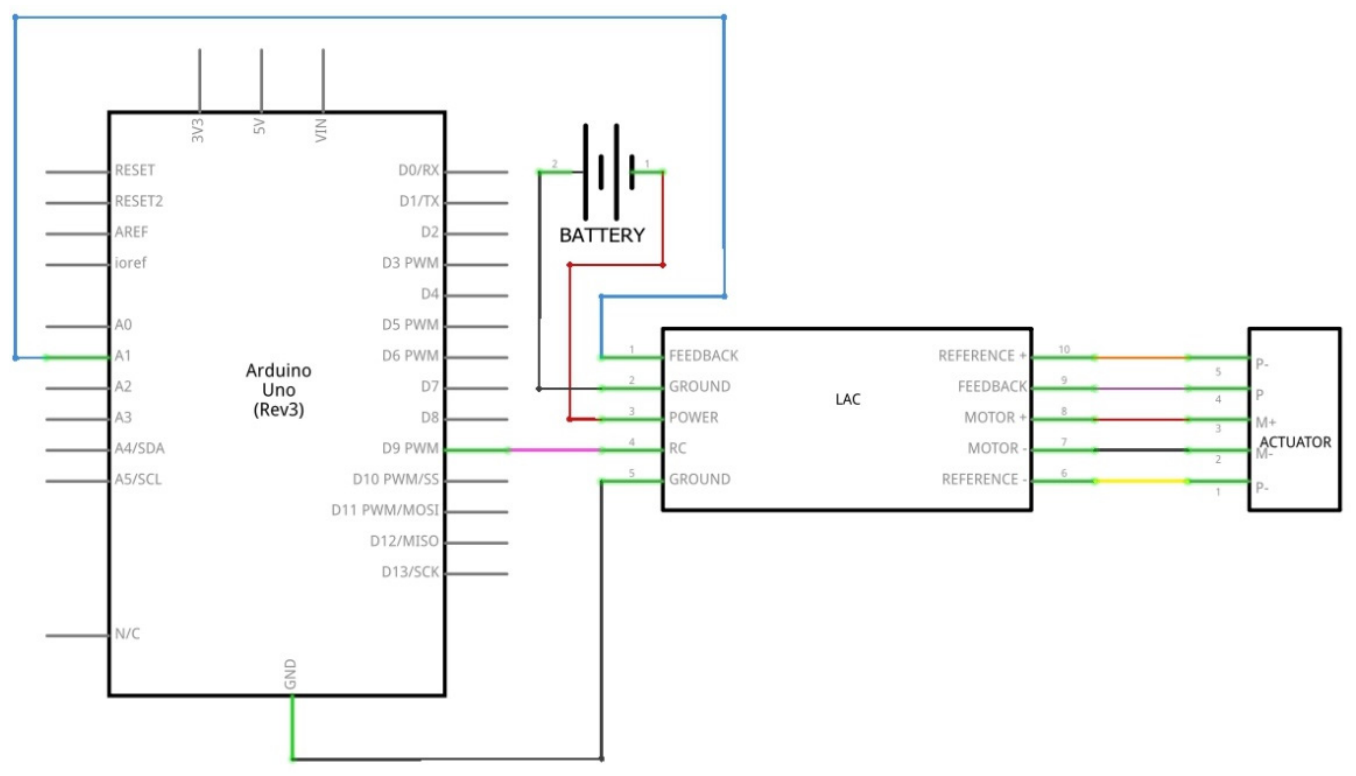

Figure 12. Arduino-LAC-motor circuit of the test bench.

\subsection{Software for the Test Bench Functioning and for Data Collection, Storage and Processing}

The Arduino development environment is used to generate the code to acquire the sensor signals and to control the actuator. The actuator is a servo motor, so the appropriate library is used and a Servo object is created.

The software, which provides the motion reference to Arduino and which collects, records and processes the data, is developed in the POWER-KI environment. POWER-KI is a programming language developed by an Italian software house for the creation of intelligent applications in the fields of IoT, Web, supervision and management systems for industrial automation [46-48]. The choice of this environment is motivated by some main features of this language: the efficiency in managing communication with different devices, the simplicity in the generation of the graphic interface and the Native Cloud (NC), i.e., the decoupling between the Graphical User Interface (GUI) and the code that generated it.

The desired position is provided by the POWER-KI software by sending a buffer of length one byte and it is read by the Arduino via the serial port, then it is used for actuator movement. The sensor bending and position feedback from the control board are read from the appropriate pins and are sent to POWER-KI via the serial port.

The "CHARACTERIZATION" software (Figure 13) is dedicated to the use of the sensor test bench. This interface allows the user to characterize a new sensor by controlling the movement of the actuator (after connecting it to the Arduino) and displaying the values collected on the screen or to see the data of a previously characterized sensor. When the software is executed, the user interface of Figure 13 appears. The "RESET" button flashes, indicating to the user that it is the first button to be pressed for characterization. In fact, the "RESET" button brings the actuator to its starting position. 


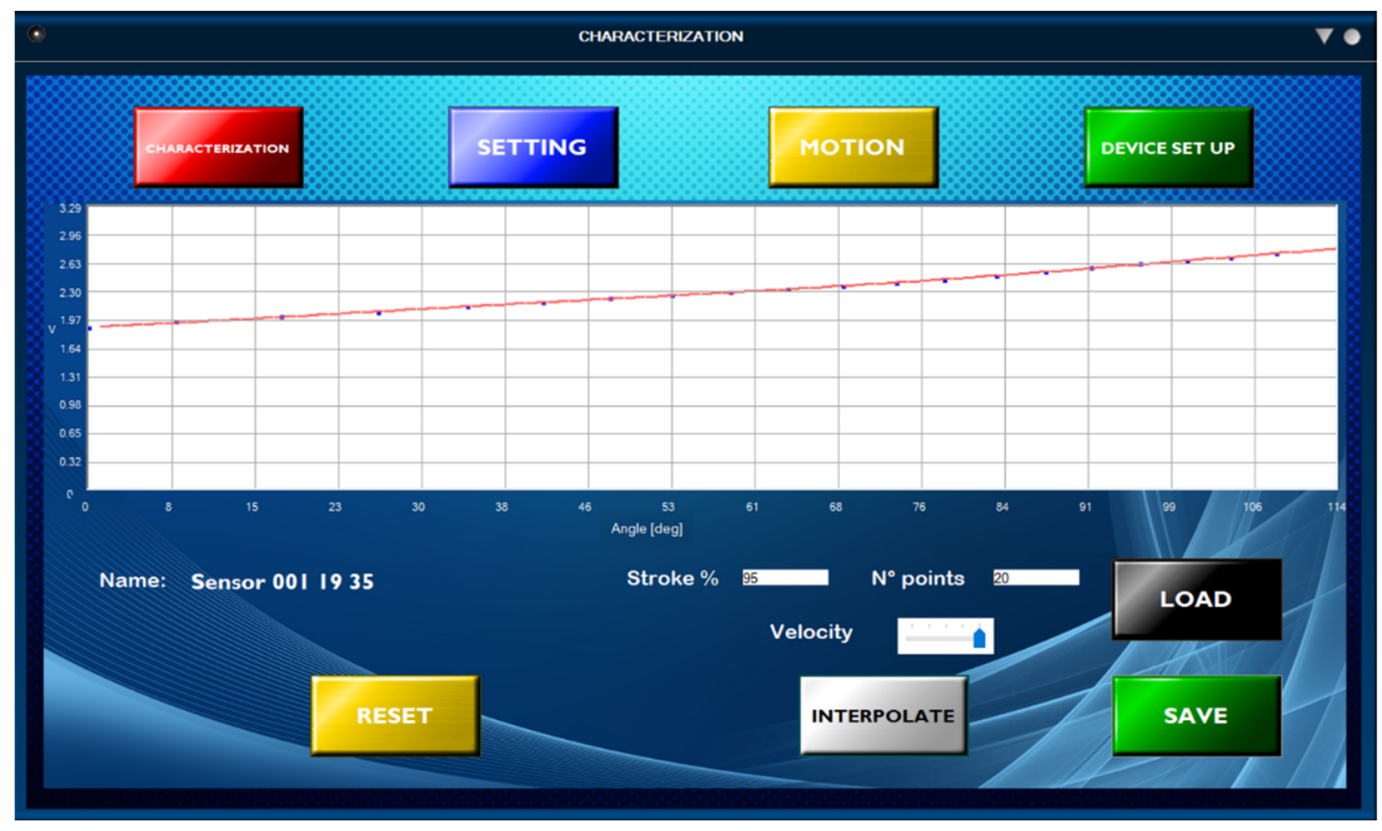

Figure 13. GUI of the software for the test bench management.

When the actuator has finished moving, the "START" button appears and the characterization begins. The user can choose the stroke of the actuator, the number of points at which it stops and acquires sensor flexion and the speed of movement of the actuator. When "START" is pressed, the actuator starts moving and stops at the first point long enough for the sensor to stabilize. Then, the sensor flexion data and motor position feedback are provided to POWER-KI by Arduino via the serial port and the real-time graph is updated with the new point recorded. At any time, the user can press the "STOP" button. In this case, the actuator stops: it is necessary to start the characterization from scratch by pressing the "RESET" button, and the graph is deleted. When the actuator has completed the characterization, it is possible to save the data by pressing the "SAVE" button: a window is opened that allows the name of the sensor that has been characterized to be entered. When the "CONFIRM" button is pressed, the characterization parameters (stroke and number of points) and the data collected are saved in a SENS type text document and named with the chosen name of the sensor. It is also possible to interpolate the various collected points and draw the interpolating function on the graph by pressing the "INTERPOLATE" button.

\section{Results}

This paragraph reports and analyses the results of experimental tests carried out on commercial flexion sensors to verify the test bench adequacy and to evaluate its performance. The tests were executed with the test bench configuration for fixed radius of curvature. Although both the configurations of the device could and should be tested for a complete evaluation on the system performance, tests were executed in this first campaign with the test bench configuration for fixed radius of curvature. This allowed for a preliminary evaluation of the main device characteristics under simplified conditions, since data are comparable per se. The motor was connected to the LAC board and to Arduino for control and data collection, which was done using the POWER-KI software. Tests were carried out using five commercial flex sensor 2.2 in length produced by Spectra Symbol. Each test involved an actuator stroke of $95 \mathrm{~mm}$ (maximum possible stroke $100 \mathrm{~mm}$ ), the collection of sensor resistance data and motor position feedback at 20 points (including the extremes at $0 \mathrm{~mm}$ and $95 \mathrm{~mm}$ positions) and a stop time between one motor movement and the next of $3 \mathrm{~s}$ to allow the actuator and sensor to stabilize. The measurements were taken at the end of this pause time and are the average value of 10 repetitions, in order to improve the accuracy of the performed tests. The measurements were performed in both 
the forward and the backward path for a total of five proofs for each sensor. The data saved during each measure are the actuator position feedback, which is read by a potentiometer inside the motor and the resistance value of the sensor. Both values are given as input to the Arduino as a voltage and are reported in a range of $0-1023$. In particular, the calculation of the resistance change of the sensor is done by means of a voltage divider, using a known resistance and an input voltage of $3.3 \mathrm{~V}$. In this case, the voltage on the sensor that is read by the Arduino is:

$$
V_{\text {out }}=V_{\text {in }} \frac{R_{s}}{R_{s}+R}
$$

In order to evaluate the best resistance to use, tests were carried out with different known resistances. Since the sensors are intended to be used to monitor the finger flexion, it is useful to know the relationship between the flexion angle of the sensor and its output. Therefore, it is necessary to calculate, for each detected motor position, the corresponding angle assumed by the sensor.

Knowing the dimensions of the designed test bench and the position of the actuator (d), the angle of interest can be calculated (Figures 14 and 15) as follows.

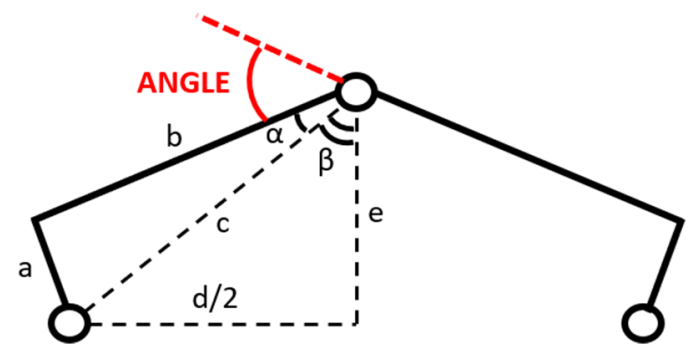

Figure 14. Angle of interest calculation.

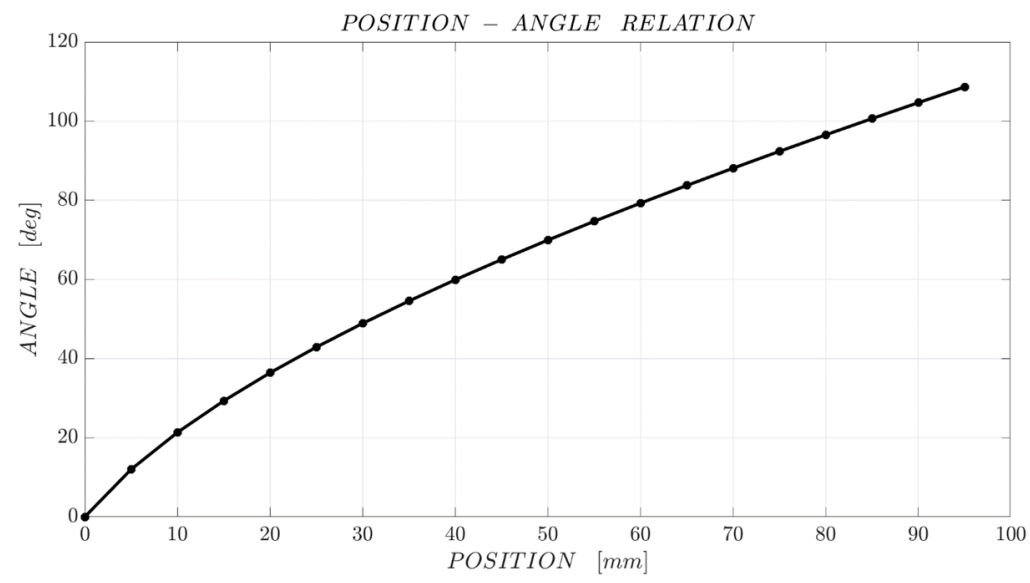

Figure 15. Position angle curve.

The angle to be calculated is the supplementary of the angle given by twice the sum of the angles $\alpha$ and $\beta$ :

$$
A N G L E=180^{\circ}-2 \cdot(\alpha+\beta)
$$

Knowing the values of $a$ and $b$, it is possible to calculate $c$ using the Pythagorean theorem:

$$
c=\sqrt{a^{2}+b^{2}}
$$

Considering now the right-angle triangle $a b c$, the angle $\alpha$ can be calculated:

$$
c \cdot \sin (\alpha)=a \rightarrow \alpha=\sin ^{-1} \frac{a}{c}
$$


Whereas, $\beta$ can be derived from the right-angle triangle $c d / 2 e$ :

$$
c \cdot \sin (\beta)=\frac{d}{2} \rightarrow \beta=\sin ^{-1} \frac{d / 2}{c}
$$

With regard to the choice of the correct resistor to insert in the circuit, Figure 16 shows the results of the testing of a sensor using resistances of $10 \mathrm{k} \Omega, 22 \mathrm{k} \Omega, 47 \mathrm{k} \Omega, 100 \mathrm{k} \Omega$ and $330 \mathrm{k} \Omega$.

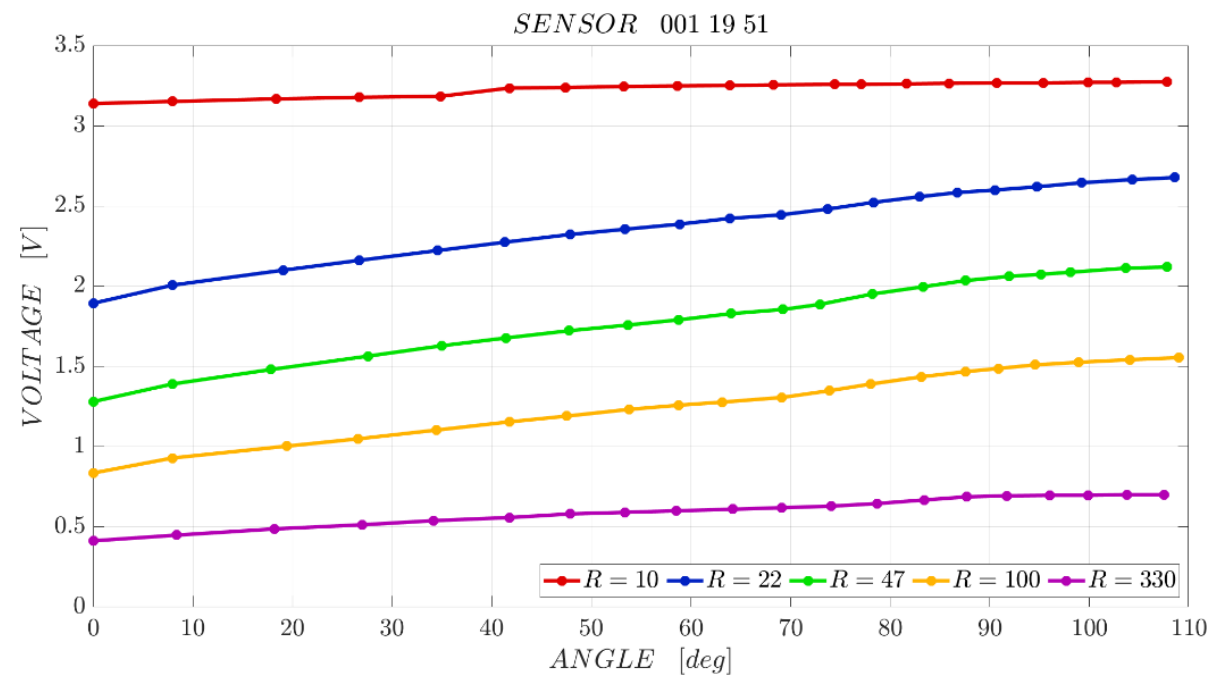

Figure 16. Example of resistance dimensioning for a sensor.

The choice of the best resistor to use within the circuit for monitoring the sensor movement is based on the comparison of the obtained characteristic curves in terms of sensitivity. To carry out this analysis, for each curve, the output range for the measuring range of interest (i.e., the difference between the highest and lowest values) and the slope of the linear regression line calculated by the method of least squares were compared, in order to find the resistance that gives the sensor the best sensitivity (as in [49]).

Considering Table $1,10 \mathrm{k} \Omega$ and $330 \mathrm{k} \Omega$ resistors can be immediately excluded, as their output range is very low, while that of the other sensors is comparable. Considering also the angular coefficient of the linear regression line of the characterization curve shown in Figure 17 and Table 2, it can be seen that the best resistance in terms of sensor sensitivity is at $47 \mathrm{k} \Omega$.

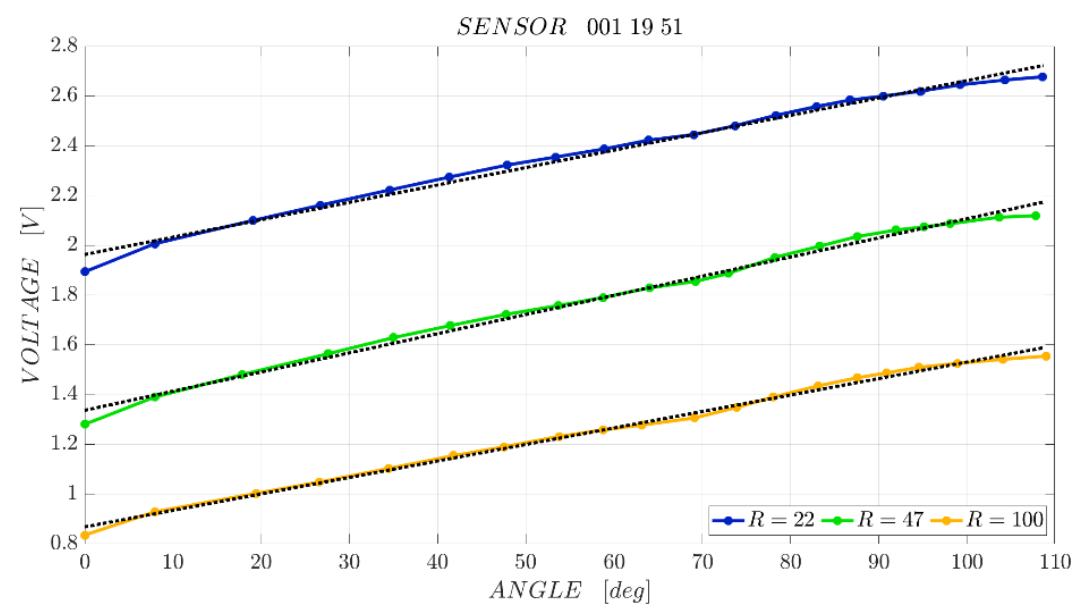

Figure 17. Analysis of resistance dimensioning: regression line of commercial sensor. 
Table 1. Analysis of resistance dimensioning: output range of commercial sensor.

\begin{tabular}{cc}
\hline$R[\mathbf{k} \Omega]$ & Output Range [V] \\
\hline 10 & 0.14 \\
22 & 0.78 \\
47 & 0.84 \\
100 & 0.72 \\
330 & 0.29 \\
\hline
\end{tabular}

Table 2. Analysis of resistance dimensioning: angular coefficient of regression line of commercial sensor.

\begin{tabular}{cc}
\hline $\boldsymbol{R}[\mathbf{k} \Omega]$ & Angular Coefficient \\
\hline 22 & 0.0070 \\
47 & 0.0077 \\
100 & 0.0066 \\
\hline
\end{tabular}

Figure 18 shows an example of characterization of a sensor, choosing a resistance of $47 \mathrm{k} \Omega$. The graphs show both the forward path data (solid lines) and the backward path data (dashed lines).

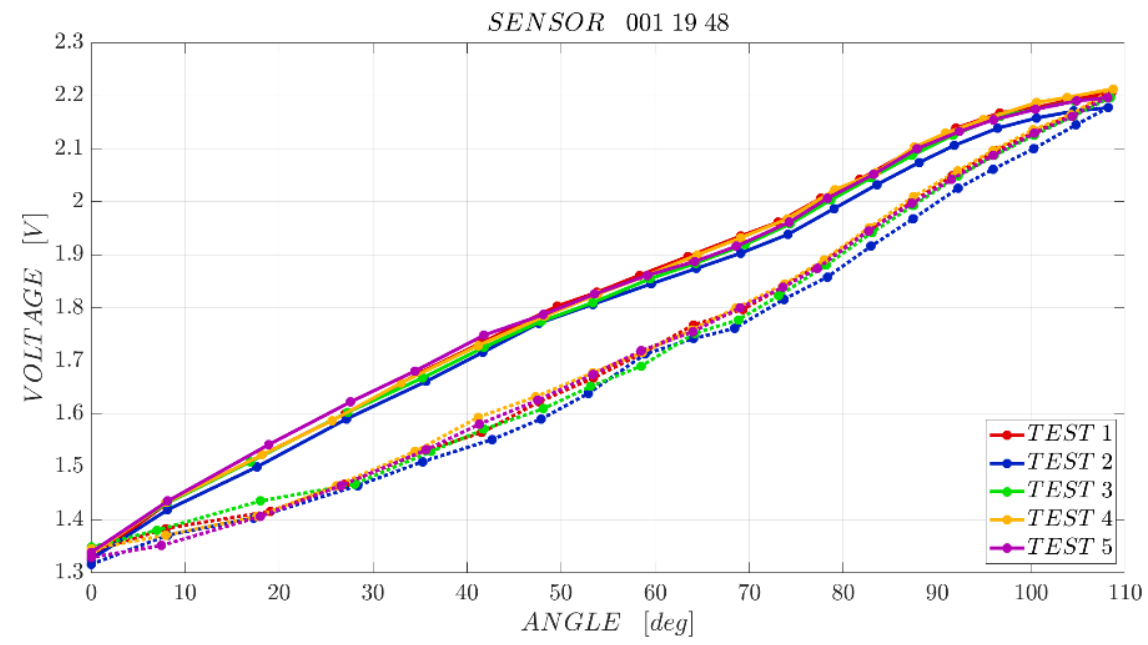

Figure 18. Example of sensor characterization with $R=47 \mathrm{k} \Omega$.

For each sensor, the hysteresis was calculated, i.e., the maximum difference between two values of the output corresponding to the same input, which is obtained during the calibration cycle for increasing and decreasing values of the input (an example is shown in Figure 19). Since theoretically also the test bench could provide a contribution to the total amount of captured hysteresis, the hysteresis phenomenon will be addressed in the following considering sensor and test bench as a whole, i.e., a sensor/test bench system.

According to the datasheet, commercial sensors have a tolerance of $30 \%$ and their resistance ranges from $25 \mathrm{k} \Omega$ to $125 \mathrm{k} \Omega$. To calculate the expected full scale output (FSO), the difference between these two values was calculated, taking into account the tolerance (thus using a range of $17.5-162.5 \mathrm{k} \Omega$ ) and reported in terms of voltage:

$$
\mathrm{FSO}=V_{\text {in }} \frac{R_{\max }}{R_{\max }+R}-V_{\text {in }} \frac{R_{\min }}{R_{\min }+R}=3.3 *\left(\frac{162.5}{162.5+47}-\frac{17.5}{17.5+47}\right)=1.66 \mathrm{~V}
$$




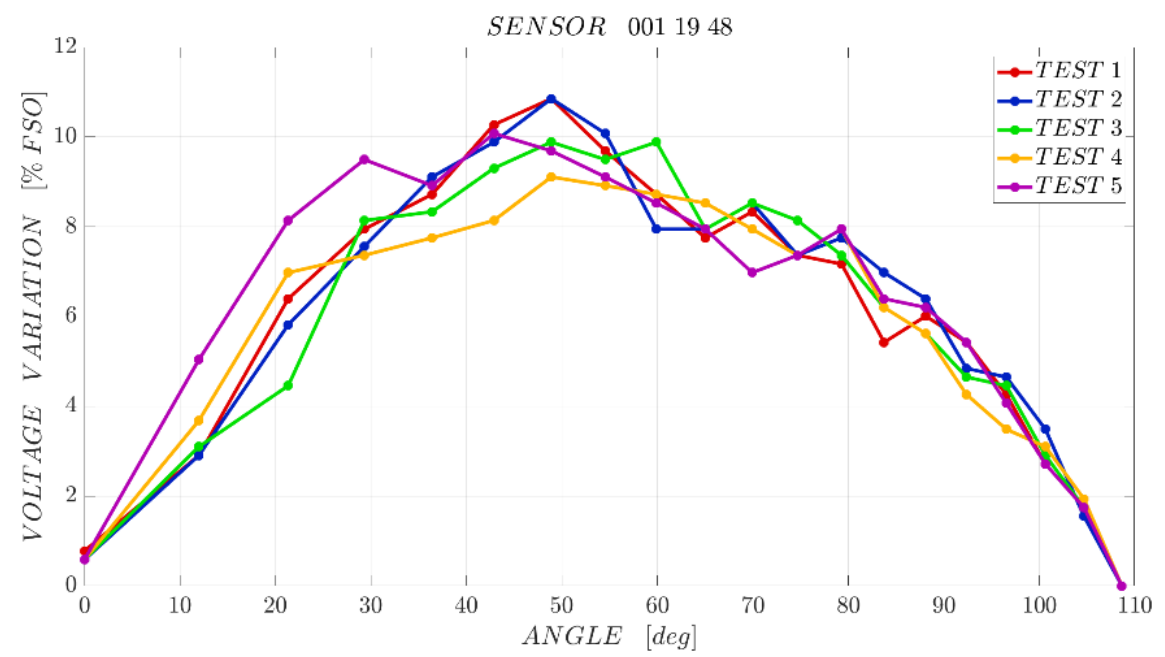

Figure 19. Example of a sensor hysteresis evaluation.

The maximum difference between the forward and backward paths of the characterization is obtained in the central part of the curves, for bending angles of approximately $40-60^{\circ}$. For all the tested sensors, the maximum hysteresis falls in the interval $11-12 \%$ of the FSO.

The repeatability of a sensor is the variability of its output when the same input value is applied. Therefore, it is the maximum difference between the outputs of two calibration cycles. For each sensor, the repeatability of the forward path and the backward path was calculated. To assess which calibration cycles differed the most, the deviation between the calibration curve and the average of all curves was calculated for each repetition. Once these curves were identified, the sensor repeatability was calculated. Figure 20 shows an example of deviation from the average curve in the backward path for a sensor in five different tests. Figure 21 reports the repeatability evaluated for the same sensor, showing the most significant comparison by comparing the most different calibration curves.

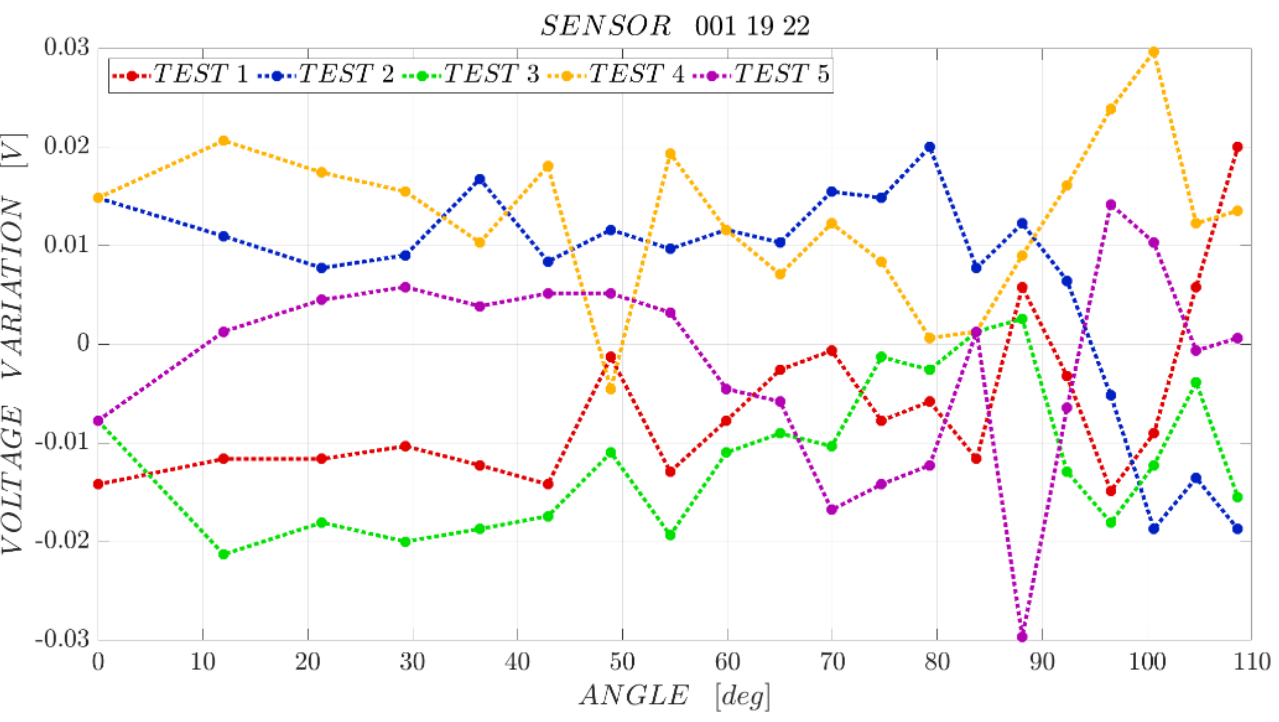

Figure 20. Sensor 00119 22: deviation from the average curve (backward path). 


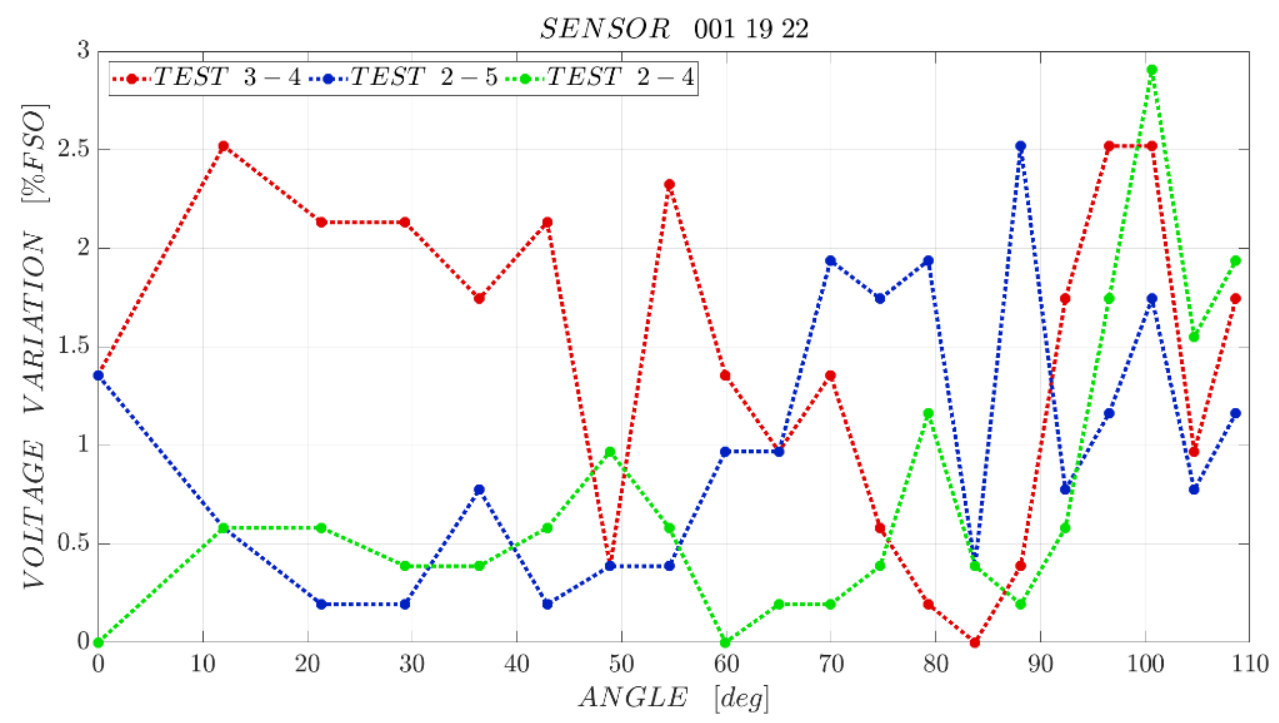

Figure 21. Sensor 001 19 22: repeatability (backward path).

The greatest deviation occurs between the curves of tests 2 and 4 . In this case the repeatability is $97.1 \%$ FSO. Overall, the repeatability of sensor "001 1922 " is 96.3\% FSO.

The linearity of a sensor is the maximum deviation of its characteristic curve from a reference line. In this case, the line that best approximates the calibration curve of the sensor, calculated by the method of least squares, was taken as the reference (Figure 22).
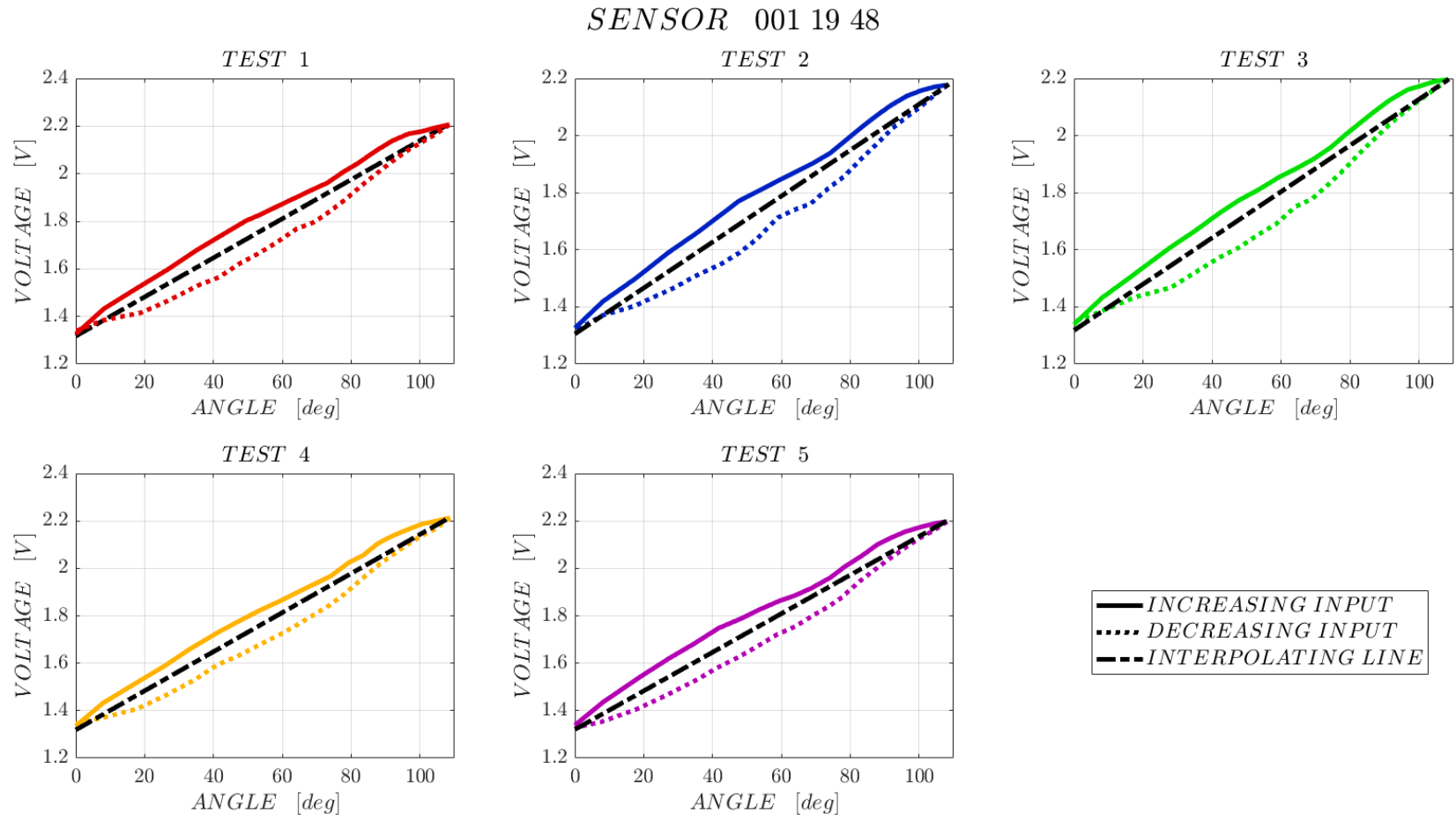

Figure 22. Reference lines for the sensor 0011948 .

For sensor "001 1948 ", the maximum deviation from the reference line is reached in the backward path of test 3 (Figure 23). The linearity of the sensor is $93.2 \%$ FSO. 


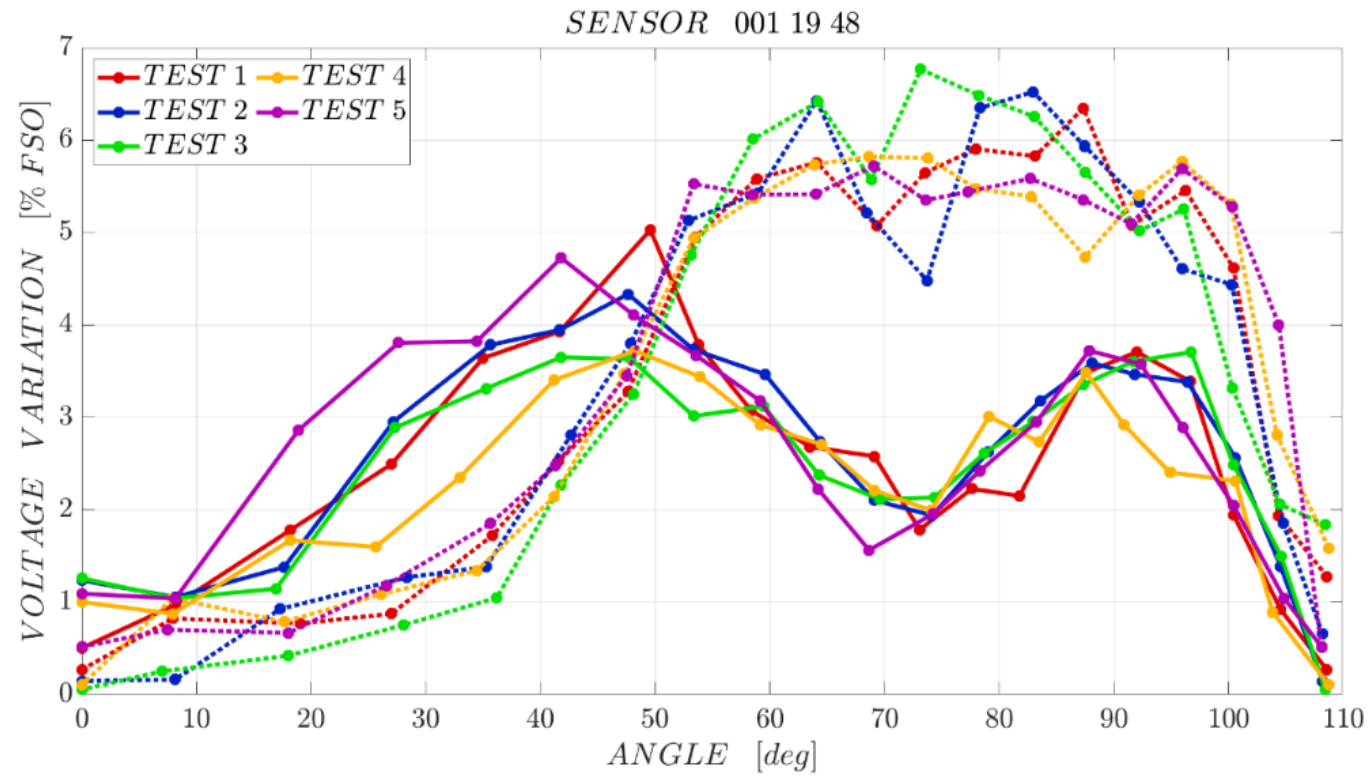

Figure 23. Linearity of the sensor 0011948.

Table 3 summarises the results of the evaluation of repeatability and linearity of the sensors and of hysteresis of the system sensor/test bench, whereas Tables 4 and 5 present the detail of mean values and standard deviation for the measured angles and voltage respectively, as detected in forward and backward paths. The boxplots of the gathered data for both the paths are also presented: Figure 24 collects them for the measured angles, whereas Figure 25 describes the voltage reference lines.

Table 3. Evaluated sensors' repeatability and linearity and sensor/test bench hysteresis.

\begin{tabular}{ccccc}
\hline Sensor & $\begin{array}{c}\text { Hysteresis } \\
{[\% \text { FSO] }}\end{array}$ & $\begin{array}{c}\text { Repeatability } \\
\text { [\% FSO] } \\
\text { Forward Path }\end{array}$ & $\begin{array}{c}\text { Repeatability } \\
\text { [\% FSO] } \\
\text { Backward Path }\end{array}$ & $\begin{array}{c}\text { Linearity } \\
\text { [\% FSO] }\end{array}$ \\
\hline 0011922 & 12.8 & 96.3 & 97.1 & 92.7 \\
0011928 & 11.8 & 96.1 & 95.7 & 93.4 \\
0011935 & 8.5 & 95.3 & 96.7 & 94.9 \\
0011948 & 10.9 & 97.5 & 97.5 & 93.2 \\
0011951 & 12.2 & 92.8 & 92 & 92.2 \\
\hline
\end{tabular}


Table 4. Mean and std of the angle values for each reached position, in the forward and backward path, for each sensor. Values in the table are expressed in degrees.

\begin{tabular}{|c|c|c|c|c|c|c|c|c|c|c|c|c|c|c|c|c|c|c|c|c|c|c|}
\hline \multirow{2}{*}{ Sensor } & \multirow{2}{*}{ Path } & & \multicolumn{20}{|c|}{ Position } \\
\hline & & & 1 & 2 & 3 & 4 & 5 & 6 & 7 & 8 & 9 & 10 & 11 & 12 & 13 & 14 & 15 & 16 & 17 & 18 & 19 & 20 \\
\hline \multirow{4}{*}{0011922} & \multirow{2}{*}{ Forw. } & mean & 0 & 7.83 & 17.0 & 25.8 & 33.5 & 41.1 & 47.9 & 53.3 & 58.6 & 63.3 & 69.1 & 74.2 & 78.1 & 83.0 & 87.4 & 91.5 & 95.7 & 99.8 & 104.0 & 108.0 \\
\hline & & std & 0 & 0.89 & 0.98 & 1.24 & 0.79 & 1.12 & 0.31 & 0.11 & 0.49 & 0.77 & 0.23 & 0.69 & 0.60 & 0.44 & 0.19 & 0.38 & 0.81 & 0.77 & 0.86 & 1.32 \\
\hline & \multirow[b]{2}{*}{ Backw. } & mean & 0 & 7.61 & 17.8 & 26.9 & 34.8 & 41.5 & 47.5 & 53.1 & 58.5 & 64.0 & 69.1 & 73.6 & 78.3 & 82.9 & 87.5 & 91.9 & 96.3 & 100.6 & 104.4 & 108.1 \\
\hline & & std & 0 & 0.68 & 0.40 & 0.64 & 0.40 & 0.19 & 0.53 & 0.29 & 0.14 & 0.25 & 0.31 & 0.29 & 0.11 & 0.14 & 0.20 & 0.44 & 0.31 & 0.04 & 0.04 & 1.32 \\
\hline \multirow{4}{*}{0011928} & \multirow{2}{*}{ Forw. } & mean & 0 & 8.02 & 18.2 & 27.0 & 34.4 & 41.5 & 48.4 & 53.9 & 59.0 & 64.0 & 69.2 & 74.1 & 78.6 & 83.5 & 87.6 & 91.8 & 96.0 & 100.6 & 104.7 & 108.75 \\
\hline & & std & 0 & 0.37 & 0.54 & 0.55 & 0.79 & 0.30 & 0.68 & 0.61 & 0.47 & 0.12 & 0.34 & 0.22 & 0.35 & 0.24 & 0.21 & 0.10 & 0.23 & 0.29 & 0.23 & 0.29 \\
\hline & \multirow[b]{2}{*}{ Backw. } & mean & 0 & 7.93 & 18.3 & 27.0 & 34.9 & 42.2 & 47.4 & 53.1 & 58.5 & 63.9 & 69.2 & 73.8 & 78.3 & 82.9 & 87.7 & 92.1 & 96.1 & 100.3 & 104.41 & 108.6 \\
\hline & & std & 0 & 0.62 & 0.75 & 0.57 & 0.78 & 0.38 & 0.18 & 0.14 & 0.31 & 0.60 & 0.25 & 0.21 & 0.18 & 0.29 & 0.45 & 0.49 & 0.20 & 0.11 & 0.15 & 0.29 \\
\hline \multirow{4}{*}{0011935} & \multirow[b]{2}{*}{ Forw. } & mean & 0 & 8.11 & 17.9 & 26.5 & 34.8 & 41.8 & 48.0 & 53.5 & 58.9 & 64.3 & 69.1 & 73.9 & 78.3 & 83.1 & 87.5 & 91.7 & 96.3 & 100.5 & 104.6 & 108.7 \\
\hline & & std & 0 & 0.39 & 0.78 & 0.86 & 0.24 & 0.36 & 0.26 & 0.28 & 0.23 & 0.41 & 0.14 & 0.36 & 0.39 & 0.24 & 0.12 & 0.21 & 0.17 & 0.24 & 0.93 & 0.19 \\
\hline & \multirow[b]{2}{*}{ Backw. } & mean & 0 & 7.80 & 17.3 & 26.4 & 34.6 & 41.2 & 47.5 & 53.2 & 58.7 & 63.6 & 69.0 & 73.9 & 78.1 & 83.0 & 87.6 & 91.9 & 96.0 & 100.38 & 104.4 & 108.7 \\
\hline & & std & 0 & 0.20 & 0.16 & 0.31 & 0.33 & 0.14 & 0.35 & 0.14 & 0.09 & 0.15 & 0.30 & 0.36 & 0.20 & 0.12 & 0.07 & 0.12 & 0.09 & 0.12 & 0.11 & 0.19 \\
\hline \multirow{4}{*}{0011948} & \multirow{2}{*}{ Forw. } & mean & 0 & 8.11 & 17.9 & 26.9 & 34.6 & 41.6 & 48.2 & 53.5 & 59.1 & 64.1 & 69.1 & 73.9 & 78.5 & 83.0 & 87.6 & 91.7 & 96.1 & 100.4 & 104.4 & 108.4 \\
\hline & & std & 0 & 0.09 & 0.72 & 0.76 & 1.05 & 0.25 & 0.75 & 0.24 & 0.48 & 0.39 & 0.33 & 0.52 & 0.61 & 0.73 & 0.28 & 0.55 & 0.76 & 0.087 & 0.38 & 0.25 \\
\hline & \multirow[b]{2}{*}{ Backw. } & mean & 0 & 7.71 & 18.0 & 27.2 & 35.4 & 41.7 & 47.5 & 53.6 & 58.7 & 64.0 & 68.8 & 73.5 & 77.9 & 82.9 & 87.4 & 91.9 & 96.8 & 100.3 & 104.4 & 108.4 \\
\hline & & std & 0 & 0.44 & 0.66 & 0.96 & 0.66 & 0.58 & 0.31 & 0.21 & 0.18 & 0.11 & 0.33 & 0.24 & 0.42 & 0.17 & 0.08 & 0.37 & 0.14 & 0.09 & 0.20 & 0.25 \\
\hline \multirow{4}{*}{0011951} & \multirow{2}{*}{ Forw. } & mean & 0 & 7.88 & 18.3 & 26.8 & 34.2 & 41.5 & 47.6 & 53.4 & 58.7 & 63.9 & 69.1 & 73.9 & 78.1 & 83.0 & 87.4 & 91.8 & 95.9 & 99.9 & 104.1 & 108.2 \\
\hline & & std & 0 & 0.66 & 1.08 & 0.91 & 1.01 & 0.43 & 0.15 & 0.20 & 0.38 & 0.12 & 0.18 & 0.58 & 0.50 & 0.22 & 0.22 & 0.06 & 0.49 & 1.06 & 0.44 & 0.62 \\
\hline & \multirow[b]{2}{*}{ Backw. } & mean & 0 & 7.97 & 17.8 & 26.5 & 34.7 & 41.5 & 47.7 & 53.2 & 58.6 & 63.8 & 68.9 & 73.7 & 78.2 & 83.0 & 87.6 & 92.1 & 96.1 & 100.2 & 104.4 & 108.2 \\
\hline & & std & 0 & 0.61 & 0.66 & 0.36 & 0.61 & 0.10 & 0.28 & 0.14 & 0.08 & 0.15 & 0.19 & 0.20 & 0.19 & 0.04 & 0.18 & 0.55 & 0.12 & 0.32 & 0.10 & 0.62 \\
\hline
\end{tabular}


Table 5. Mean and std of the voltage values for each reached position, in the forward and backward path, for each sensor. Values in the table are expressed in $V$.

\begin{tabular}{|c|c|c|c|c|c|c|c|c|c|c|c|c|c|c|c|c|c|c|c|c|c|c|}
\hline \multirow{2}{*}{ Sensor } & \multirow{2}{*}{ Path } & & \multicolumn{20}{|c|}{ Position } \\
\hline & & & 1 & 2 & 3 & 4 & 5 & 6 & 7 & 8 & 9 & 10 & 11 & 12 & 13 & 14 & 15 & 16 & 17 & 18 & 19 & 20 \\
\hline \multirow{3}{*}{0011922} & \multirow{2}{*}{ Forw. } & mean & 1.44 & 1.59 & 1.71 & 1.80 & 1.88 & 1.95 & 2.01 & 2.04 & 2.07 & 2.10 & 2.13 & 2.17 & 2.19 & 2.23 & 2.27 & 2.29 & 2.31 & 2.33 & 2.34 & 2.35 \\
\hline & & std & 0.01 & 0.01 & 0.02 & 0.02 & 0.02 & 0.02 & 0.01 & 0.01 & 0.02 & 0.02 & 0.01 & 0.02 & 0.02 & 0.02 & 0.02 & 0.01 & 0.02 & 0.01 & 0.01 & 0.01 \\
\hline & Backw. & std & 0.02 & 0.01 & 0.02 & 0.02 & 0.01 & 0.02 & 0.01 & 0.01 & 0.01 & 0.01 & 0.01 & 0.01 & 0.02 & 0.01 & 0.02 & 0.02 & 0.01 & 0.01 & 0.02 & 0.01 \\
\hline \multirow{3}{*}{0011928} & \multirow{3}{*}{$\begin{array}{l}\text { Forw. } \\
\text { Backw. }\end{array}$} & mean & 1.27 & 1.36 & 1.45 & 1.53 & 1.59 & 1.65 & 1.71 & 1.74 & 1.77 & 1.80 & 1.83 & 1.86 & 1.89 & 1.93 & 1.96 & 1.98 & 2.01 & 2.03 & 2.04 & 2.06 \\
\hline & & std & 0.00 & 0.01 & 0.01 & 0.01 & 0.01 & 0.02 & 0.02 & 0.02 & 0.02 & 0.01 & 0.01 & 0.01 & 0.01 & 0.02 & 0.02 & 0.02 & 0.02 & 0.02 & 0.02 & 0.02 \\
\hline & & mean & 1.28 & 1.31 & 1.34 & 1.39 & 1.44 & 1.49 & 1.53 & 1.57 & 1.61 & 1.65 & 1.69 & 1.73 & 1.77 & 1.81 & 1.86 & 1.90 & 1.94 & 1.98 & 2.01 & 2.06 \\
\hline \multirow{4}{*}{0011935} & \multirow{4}{*}{$\begin{array}{l}\text { Forw. } \\
\text { Backw. }\end{array}$} & mean & 1.33 & 1.39 & 1.45 & 1.51 & 1.56 & 1.60 & 1.64 & 1.66 & 1.69 & 1.72 & 1.73 & 1.76 & 1.79 & 1.82 & 1.85 & 1.87 & 1.90 & 1.92 & 1.93 & 1.95 \\
\hline & & std & 0.01 & 0.02 & 0.01 & 0.02 & 0.02 & 0.03 & 0.02 & 0.03 & 0.03 & 0.03 & 0.03 & 0.03 & 0.03 & 0.03 & 0.03 & 0.03 & 0.03 & 0.02 & 0.02 & 0.02 \\
\hline & & mean & 1.33 & 1.36 & 1.38 & 1.41 & 1.44 & 1.48 & 1.51 & 1.54 & 1.57 & 1.59 & 1.62 & 1.65 & 1.67 & 1.71 & 1.75 & 1.79 & 1.83 & 1.86 & 1.90 & 1.95 \\
\hline & & std & 0.02 & 0.01 & 0.02 & 0.02 & 0.01 & 0.02 & 0.01 & 0.01 & 0.01 & 0.01 & 0.01 & 0.01 & 0.02 & 0.01 & 0.02 & 0.02 & 0.01 & 0.01 & 0.02 & 0.01 \\
\hline \multirow{4}{*}{0011948} & \multirow{4}{*}{$\begin{array}{l}\text { Forw. } \\
\text { Backw. }\end{array}$} & mean & 1.33 & 1.43 & 1.52 & 1.60 & 1.67 & 1.73 & 1.78 & 1.82 & 1.86 & 1.89 & 1.92 & 1.96 & 2.01 & 2.05 & 2.09 & 2.13 & 2.16 & 2.17 & 2.19 & 2.20 \\
\hline & & std & 0.01 & 0.01 & 0.02 & 0.01 & 0.01 & 0.01 & 0.01 & 0.01 & 0.01 & 0.01 & 0.01 & 0.01 & 0.01 & 0.01 & 0.01 & 0.01 & 0.01 & 0.01 & 0.01 & 0.01 \\
\hline & & mean & 1.34 & 1.37 & 1.41 & 1.47 & 1.53 & 1.57 & 1.62 & 1.66 & 1.71 & 1.75 & 1.79 & 1.83 & 1.88 & 1.94 & 1.99 & 2.04 & 2.09 & 2.12 & 2.16 & 2.20 \\
\hline & & std & 0.02 & 0.01 & 0.02 & 0.02 & 0.01 & 0.02 & 0.01 & 0.01 & 0.01 & 0.01 & 0.01 & 0.01 & 0.02 & 0.01 & 0.02 & 0.02 & 0.01 & 0.01 & 0.02 & 0.01 \\
\hline \multirow{2}{*}{0011951} & Forw. & mean & 1.28 & 1.38 & 1.47 & 1.54 & 1.60 & 1.66 & 1.70 & 1.75 & 1.78 & 1.82 & 1.85 & 1.89 & 1.94 & 1.99 & 2.03 & 2.06 & 2.08 & 2.10 & 2.12 & 2.13 \\
\hline & Backw. & std & 0.02 & 0.01 & 0.02 & 0.02 & 0.01 & 0.02 & 0.01 & 0.01 & 0.01 & 0.01 & 0.01 & 0.01 & 0.02 & 0.01 & 0.02 & 0.02 & 0.01 & 0.01 & 0.02 & 0.01 \\
\hline
\end{tabular}



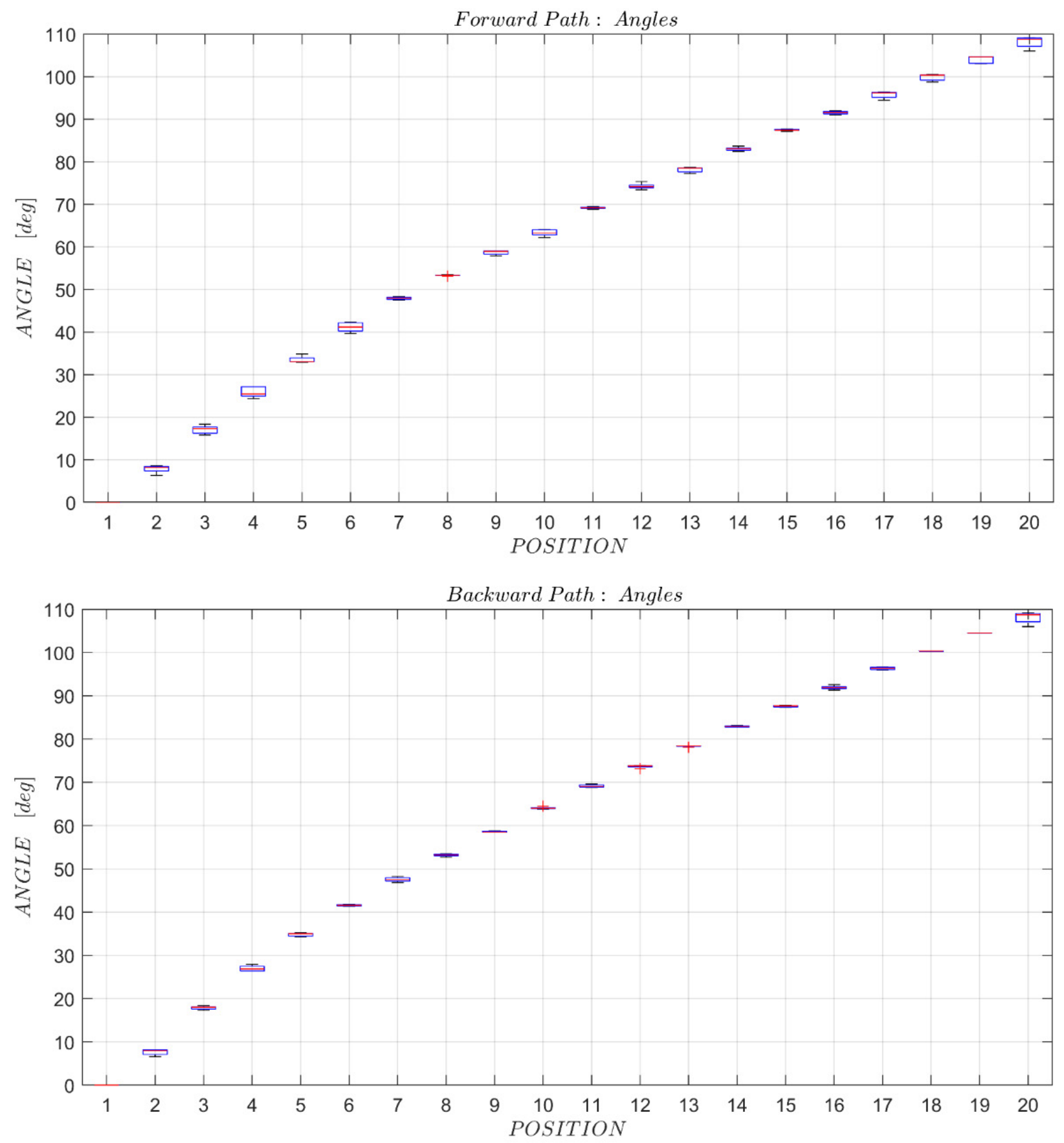

Figure 24. Boxplots of the angle values in the forward and backward paths for the sensor 0011922. 

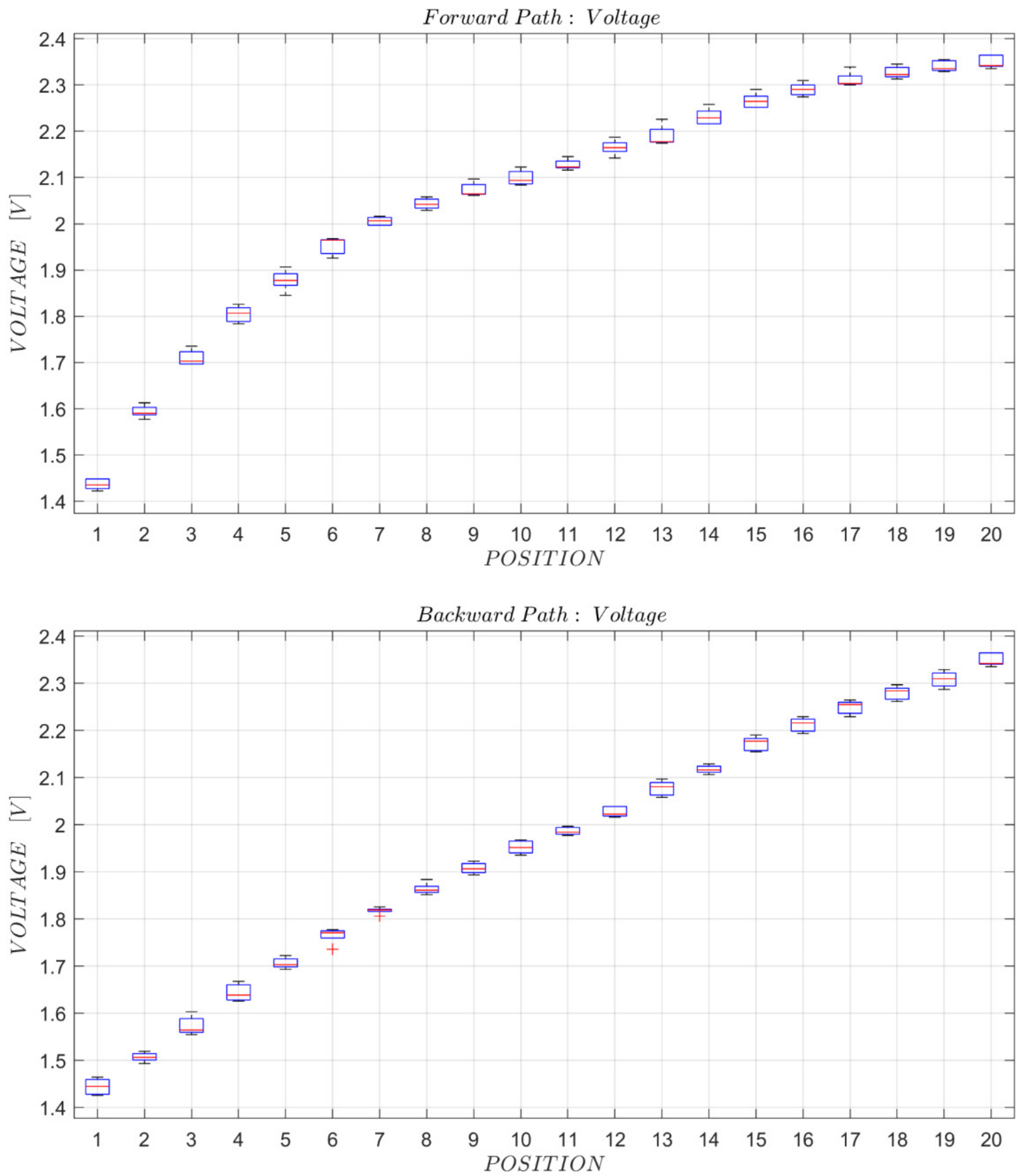

Figure 25. Reference lines for the sensor 0011922.

To compare the device behaviour in the forward and backward paths in terms of position hysteresis, two-sample $t$-tests were done, for each reached position, to verify the null hypothesis of data gathered from two populations with Equal Means, at the 5\% significance level.

The h- and $p$-values of the $t$-test for each position are collected in Table 6 . 
Table 6. Null hypothesis results (h) and $p$ values for the two-sample $t$-test of Equal Means, at the $5 \%$ significance level, in the forward and backward paths.

\begin{tabular}{|c|c|c|c|c|c|c|c|c|c|c|c|c|c|c|c|c|c|c|c|c|c|}
\hline \multirow{2}{*}{ Sensor } & & \multicolumn{20}{|c|}{ Position } \\
\hline & & 1 & 2 & 3 & 4 & 5 & 6 & 7 & 8 & 9 & 10 & 11 & 12 & 13 & 14 & 15 & 16 & 17 & 18 & 19 & 20 \\
\hline 001 & $\mathrm{~h}$ & 0 & 0 & 0 & 0 & 1 & 0 & 0 & 0 & 0 & 0 & 0 & 0 & 0 & 0 & 0 & 0 & 0 & 0 & 0 & 0 \\
\hline 1922 & $p$ & 1.00 & 0.67 & 0.13 & 0.12 & 0.01 & 0.45 & 0.19 & 0.31 & 0.55 & 0.08 & 0.83 & 0.12 & 0.46 & 0.75 & 0.32 & 0.20 & 0.17 & 0.22 & 0.26 & 1.00 \\
\hline 001 & $\mathrm{~h}$ & 0 & 0 & 0 & 0 & 0 & 1 & 1 & 1 & 0 & 0 & 0 & 0 & 0 & 1 & 0 & 0 & 0 & 0 & 0 & 0 \\
\hline 1928 & $p$ & 1.00 & 0.79 & 0.87 & 0.87 & 0.33 & 0.02 & 0.01 & 0.03 & 0.07 & 0.78 & 0.92 & 0.16 & 0.15 & 0.01 & 0.57 & 0.20 & 0.66 & 0.08 & 0.07 & 1.00 \\
\hline 001 & $\mathrm{~h}$ & 0 & 0 & 0 & 0 & 0 & 1 & 1 & 1 & 0 & 1 & 0 & 0 & 0 & 0 & 0 & 0 & 1 & 0 & 0 & 0 \\
\hline 1935 & $p$ & 1.00 & 0.16 & 0.13 & 0.84 & 0.49 & 0.01 & 0.02 & 0.04 & 0.08 & 0.01 & 0.46 & 1.00 & 0.28 & 0.50 & 0.61 & 0.06 & 0.04 & 0.23 & 0.69 & 1.00 \\
\hline 001 & $\mathrm{~h}$ & 0 & 0 & 0 & 0 & 0 & 0 & 0 & 1 & 0 & 0 & 0 & 0 & 0 & 0 & 0 & 0 & 0 & 1 & 0 & 0 \\
\hline 1948 & $p$ & 1.00 & 0.09 & 0.94 & 0.64 & 0.18 & 0.81 & 0.14 & 0.05 & 0.10 & 0.54 & 0.24 & 0.14 & 0.11 & 0.85 & 0.08 & 0.49 & 0.75 & 0.01 & 0.88 & 1.00 \\
\hline 001 & $\mathrm{~h}$ & 0 & 0 & 0 & 0 & 0 & 0 & 0 & 0 & 0 & 0 & 0 & 0 & 0 & 0 & 0 & 0 & 0 & 0 & 0 & 0 \\
\hline 1951 & $p$ & 1.00 & 0.83 & 0.45 & 0.60 & 0.43 & 0.90 & 0.55 & 0.16 & 0.57 & 0.83 & 0.09 & 0.61 & 0.72 & 0.75 & 0.39 & 0.29 & 0.42 & 0.54 & 0.14 & 1.00 \\
\hline
\end{tabular}

\section{Discussion}

\subsection{Literature Overview on Flex Sensors' Characterization}

Electrical quantitative characterization of RFSs is necessary as manufacturers generally do not provide it. In literature different methods have been referred to which are classifiable in manual, partially automated and fully automatized. A brief overview of the main solutions adopted is reported in this subsection.

Manual methods are based on different approaches: in [50] the sensor is fixed entirely on an angle gauge by a clamp; in [49] a traditional goniometer is used; in [10] the flex sensor is wrapped around a cylinder with a fixed diameter; in [3] a wooden made frame with a manual goniometer as a reference is used.

Partially automated approaches are described: in [28] where the actual bending angle during the calibration is detected by using an external electromagnetic sensing positioning system, considered as the ground truth (GT); in [44] a 3D printed mechanical testbed is used, with three different diameters, with an IMU and an incremental encoder. Fully automated solutions are described in [51] with a hinged joint that is able to rotate through a stepper motor which actuates one wing with respect to the other which is fix constrained, for the characterization with fixed radius of curvature; in [43] a pushed/pulled sliding trolley on a linear guide moved by a stepper motor allows variable radius of curvature characterization.

Table 7 collects the articles mentioned above, highlighting tools, methods and results in a synthetic way, as regards the characterization of the sensors. 


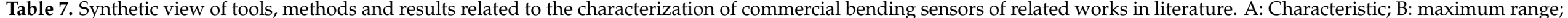
C: linearity; D: resolution; E: repeatability; F: accuracy; G: sensitivity; H: hysteresis.

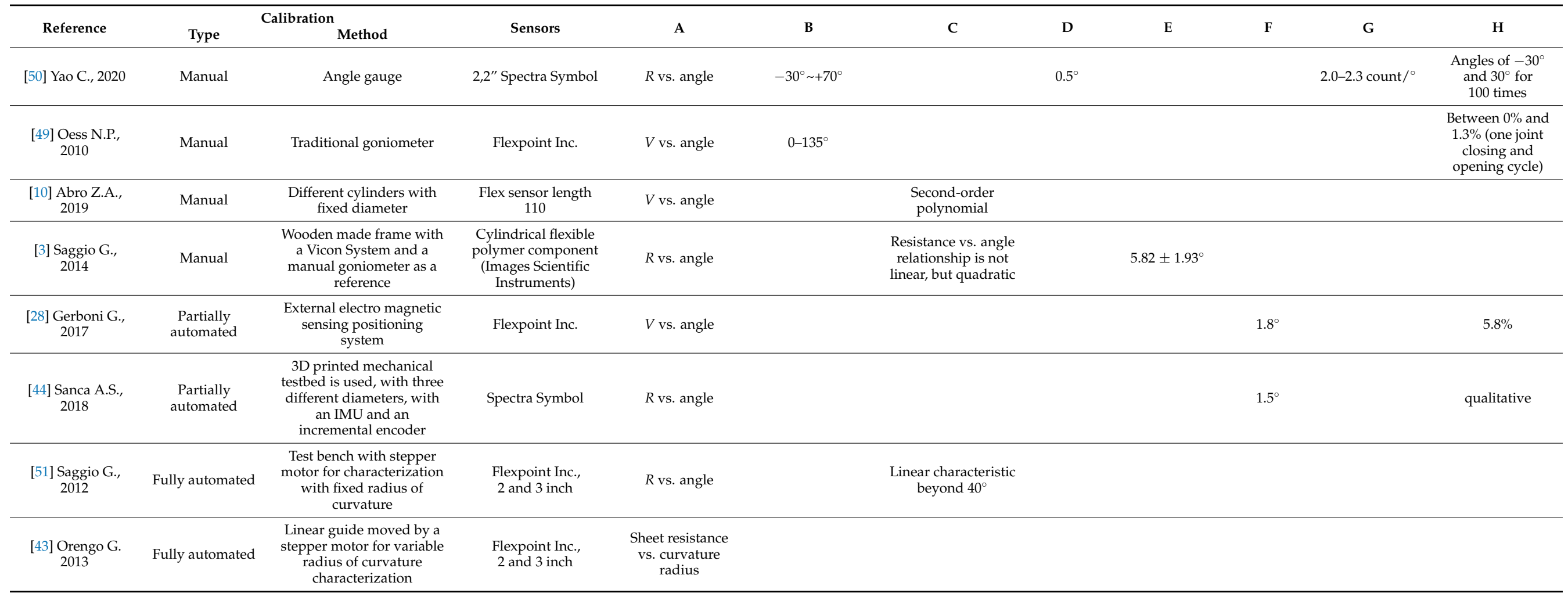




\subsection{Results Analysis}

The maximum difference between the forward and backward paths of the characterization is obtained in the central part of the curves, for bending angles of approximately 40-60". For sensors "001 $1928^{\circ}$, “001 1948 ", “001 19 51" the sensor/test bench hysteresis is around $11-12 \%$ of the full scale output. Sensor "001 1922 " has a similar behaviour, but in test 3 the hysteresis is close to $13 \%$ FSO, whereas with sensor "001 19 35" the lowest hysteresis is achieved: $8.5 \%$ FSO.

The repeatability of the tested sensors is quite high, and it is around $95-96 \%$ of the full scale output for sensors "001 19 22", "001 19 28" and "001 19 35". The "001 1948 " sensor has a higher repeatability than the others, 97.5\% FSO, whereas the "001 19 51" sensor has the lowest: $92 \%$ FSO.

The linearity of the tested commercial sensors is around $93 \%$ of the full scale output, except for the "001 19 51" sensor for which it is a little lower and the "001 19 35" sensor which has a linearity value of almost $95 \%$ FSO.

The realized device therefore allows a rapid and effective characterization of the sensors and in addition to the characteristic curve, it also allows estimating in a simple and easy way repeatability, linearity and hysteresis.

Considering these results, a preliminary validation is confirmed, based on the comparison between the results obtained on the commercial tested sensors (in terms of linearity and repeatability) and values referred to in literature [1,42] for sensors with the same constructive characteristics. Instead, a comparison with data provided by the manufacturers is not possible, since the datasheets of the components do not report the linearity and repeatability values. Focusing on the hysteresis, neither characterization results in literature nor datasheets have been found to compare with from this point of view. Therefore, it can be said that the extrapolation of sensor hysteresis from experimental tests represents an original aspect of the work. On the other hand, it is therefore not possible to obtain a preliminary validation of the goodness of the test bench for the estimation of this characteristic.

In order to be able to use the sensors, it is necessary to have a single interpolating curve that approximates well the forward and backward curves of all the tests done. For this reason, for each sensor, the average of the forward curves of each test and the average of the return curves were calculated. An example for a sensor is shown in Figure 26.

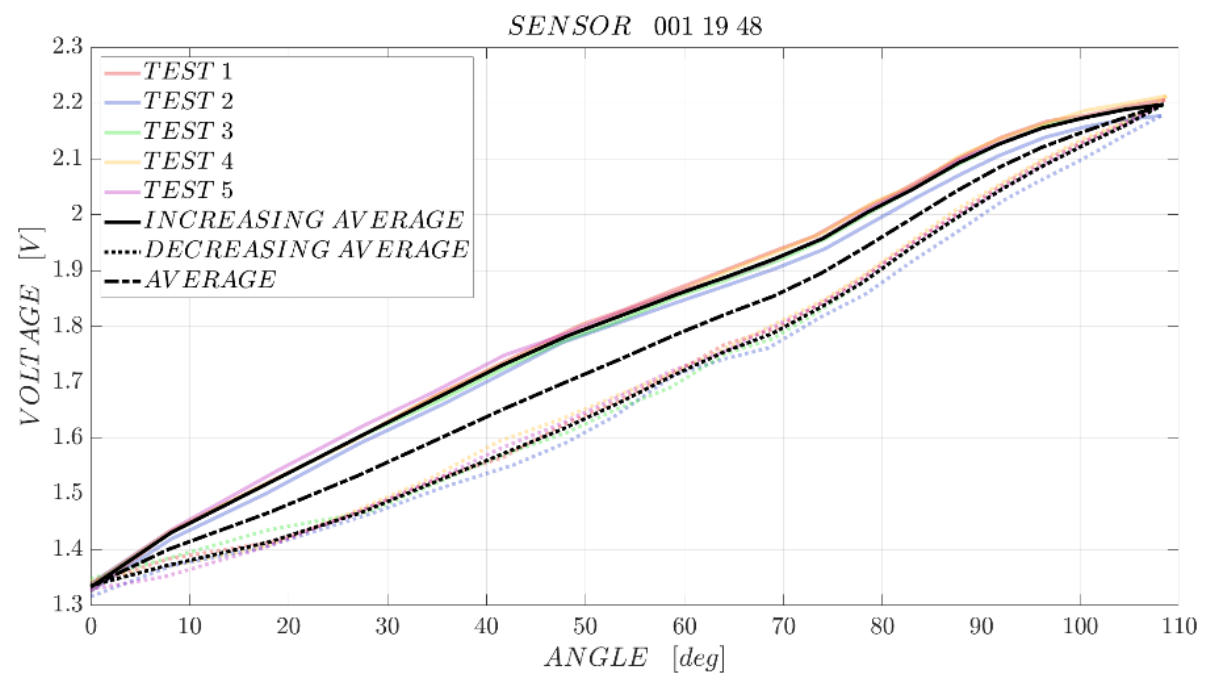

Figure 26. Sensor 00119 48: interpolation curve.

From the results of the statistical analysis of the angle values distribution in different repetitions, it emerged that the maximum standard deviation is $1.32^{\circ}$ (about $1.2 \%$ of the maximum angle value) for both the forward and backward path. This result is very positive, 
as it reveals that the repeatability of positioning is good and it is absolutely adequate for most applications for which bending sensors are normally used.

The null hypothesis of the $t$-tests to compare the angle mean values between the forward and backward path in each position was verified in most cases; in fact, it is not verified only in 12 positions out of 100 . The $p$-values are quite good, and overall it can be said that the device movement does not have a significant hysteresis between the forward and backward path.

As can be seen in Table 5, the maximum standard deviation in the voltage measurement is $0.0439 \mathrm{~V}$ (about $2 \%$ of the maximum voltage value) for the forward path and $0.0198 \mathrm{~V}$ for the backward one. For the voltage the measures dispersion is higher than for the angle. It is a reasonable result, since the dispersion on the angle also propagates on the voltage measurement; however, it is a relatively low value.

\section{Conclusions}

Based on the literature review on flexion sensor characterization (Section 4.1), the developed test bench has features that make it unique and innovative.

Manual or partially automated characterization solutions are inaccurate and require time-consuming procedures. Few fully automatic devices are reported in literature and not one allows a characterization both for fixed and variable radius of curvature.

Instead, the presented device can be theoretically used for characterizing sensors with fixed and variable radius of curvature. The case of sensors used with fixed radius of curvature was tested, whereas the variable radius case will be the object of a future work. Furthermore, the device can mount different types of sensors, by changing the geometry of the sensor carrier sheet and obtaining it quickly with 3D printing. In principle, given the kinematics of the system, the device could be also used for the characterization of deformation sensors, once it provides the proper modifications to the support foil and to the programming. This functionality is also not possible in the automatic bending sensor characterization systems described in the literature. The operations of repetition of the tests, of acquisition and of subsequent reprocessing of the results are automated and can be carried out with significant rapidity. The bench can be reproduced quickly and at a significantly lower cost.

The performed characterization of commercial sensors has obtained results that are absolutely consistent with the performance of these sensors in terms of linearity and repeatability, and this is a good indicator of the suitability of the test bench for the purpose for which it was designed and built. Instead, the execution of the hysteresis characterization of the sensors is an original contribution of the work. Nevertheless, a rigorous validation of the device is still needed, to state this certainly.

The statistical analysis of the angle values distribution confirms a good repeatability of the positioning and a very low hysteresis between the forward and the backward path.

The use of the test bench also made it possible to verify its significant simplicity of use and the speed in replacing the sensors and carrying out tests on each sensor.

As it can be seen from Table 7, in literature there are few works relating to the characterization of bending sensors. Hence, the development of an automatic system that allows an effective and rapid characterization represents an important contribution to improve the state of the art on this issue.

With reference to the constructive aspects of the test bench, the choice of the material that constitutes the foil for the positioning of the sensors and its geometry are very important for a correct characterization of the sensors. In particular, for the case of a fixed radius of curvature, an excessive stiffness of the sheet due to the material or an excessive thickness can cause a gap between the sheet and the V-shaped structure (Figure 8). This raises two major issues: first, the radius for this application may depend on the lamina material and form factors as well as the sensor itself and may differ from the radius predefined from the test-bench (V-structure) design, and second, this implementation can introduce inherent hysteresis that can superimpose hysteresis of the sensor itself. For this reason, within the 
article the detected hysteresis values have been referred to in the sensor/test bench system and not in the sensor itself. Further investigation work must be carried out to identify the possible hysteresis contribution introduced by the test bench and to reduce it if it proves to be significant. Furthermore, the support foil should be changed to use the test bench for the characterization of strain sensors. In fact, compared to those described in the article, a change in the material and in the fixing method should be envisaged. The new/specific support foil could be, for example, made of silicone rubber, with an adequate hardness value, and it is reasonable to expect that this aspect could represent the most sensitive issue to obtain adequate elongations. In fact, the maximum strokes that can be obtained will mainly be determined by the characteristics of the foil material, as the actuator has a significant stroke and therefore does not constitute a limitation.

Future developments of the presented work are: validation of the device, testing for the characterization of sensors used with variable radius of curvature, adaptation of the device to the characterization of extensible sensors, testing of the device on sensors with geometry and structural characteristics different from those considered, testing of the device for the characterization of sensors actually used in a biomedical device to verify its effectiveness in the field, implementation of functions in the software that automate all the analysis operations of the performed measurements and finding a solution to the problem of the alignment of the lamina with the V-structure previously described, possibly inserting elastic elements (springs) to keep the lamina close to the rigid V-structure.

Author Contributions: Conceptualization, M.T., A.F., C.A. and D.V.; methodology, M.T., A.F., C.A. and D.V.; software, A.F. and M.T.; validation M.T, D.V. and C.A.; formal analysis, M.T., C.A. and A.F.; data curation, A.F., M.T. and D.V.; writing-original draft preparation, M.T. and A.F.; writing-review and editing, M.T., A.F., C.A. and D.V. All authors have read and agreed to the published version of the manuscript.

Funding: This research was funded by DIMI, Department of Mechanical and Industrial Engineering, University of Brescia, within the project "AIMAREC", 2019-2021.

Institutional Review Board Statement: Not applicable.

Informed Consent Statement: Not applicable.

Data Availability Statement: Not applicable.

Conflicts of Interest: The authors declare no conflict of interest. The funders had no role in the design of the study; in the collection, analyses, or interpretation of data; in the writing of the manuscript, or in the decision to publish the results.

\section{References}

1. Saggio, G.; Riillo, F.; Sbernini, L.; Quitadamo, L.R. Resistive flex sensors: A survey. Smart Mater. Struct. 2015, $25,013001$. [CrossRef]

2. Grisbrook, T.; Imms, C.; Walmsley, C.P.; Campbell, A.; Elliott, C.; Williams, S.A. Measurement of Upper Limb Range of Motion Using Wearable Sensors: A Systematic Review. Sport. Med. Open 2018, 4, 53.

3. Saggio, G.; Quitadamo, L.R.; Albero, L. Development and evaluation of a novel low-cost sensor-based knee flexion angle measurement system. Knee 2014, 21, 896-901. [CrossRef] [PubMed]

4. Liou, J.-C.; Fang, K.-W. Flex Sensor for Stroke Patients Identify the Specific Behavior with Different Bending Situations. In Proceedings of the 2017 6th International Symposium on Next Generation Electronics, ISNE 2017, Keelung, Taiwan, 23-25 May 2017.

5. Cafolla, D.; Chen, I.M.; Ceccarelli, M. An experimental characterization of human torso motion. Front. Mech. Eng. 2015, 10, 311-325. [CrossRef]

6. Garda, Y.R.; Caesarendra, W.; Tjahjowidodo, T.; Turnip, A.; Wahyudati, S.; Nurhasanah, L.; Sutopo, D. Flex Sensor Based Biofeedback Monitoring for Post-Stroke Fingers Myopathy Patients. J. Phys. Conf. Ser. 2018, 1007, 012069. [CrossRef]

7. Habibi, A.; Nugroho, S.M.S.; Purnama, I.K.E.; Prawitri, Y.D.; Subadi, I. Health Level Classification of Motor Stroke Patients Based on Flex Sensor Using Fuzzy Logic Method. In Proceedings of the 2019 International Conference on Computer Engineering, Network, and Intelligent Multimedia, CENIM 2019, Surabaya, Indonesia, 19-20 November 2019.

8. Lakshmi, K.J.; Muneshwar, A.; Ratnam, A.V.; Kodali, P. Patient Assistance Using Flex Sensor. In Proceedings of the 2020 IEEE International Conference on Communication and Signal Processing, ICCSP 2020, Chennai, India, 28-30 July 2020 ; pp. 181-185. 
9. Guo, Y.-R.; Zhang, X.-C.; An, N. Monitoring Neck Posture with Flex Sensors. In Proceedings of the 9th International Conference on Information Science and Technology, ICIST 2019, Hulunbuir, China, 2-5 August 2019; pp. 459-463.

10. Abro, Z.A.; Yi-Fan, Z.; Nan-Liang, C.; Cheng-Yu, H.; Lakho, R.A.; Halepoto, H. A novel flex sensor-based flexible smart garment for monitoring body postures. J. Ind. Text. 2019, 49, 262-274. [CrossRef]

11. Oess, N.P.; Wanek, J.; Curt, A. Design and evaluation of a low-cost instrumented glove for hand function assessment. J. Neuroeng. Rehabil. 2012, 9, 2. [CrossRef] [PubMed]

12. Roy, K.; Idiwal, D.P.; Agrawal, A.; Hazra, B. Flex Sensor Based Wearable Gloves for Robotic Gripper Control. In Proceedings of the ACM International Conference Proceeding Series, Goa, India, 2-4 July 2015.

13. Dhepekar, P.; Adhav, Y.G. Wireless Robotic Hand for Remote Operations Using Flex Sensor. In Proceedings of the 2016 International Conference on Automatic Control and Dynamic Optimization Techniques (ICACDOT), Pune, India, 9-10 September 2016; pp. 114-118.

14. Sandesh, R.S.; Venkatesan, N. Novel Approach to Control of Robotic Hand Using Flex Sensors. IAES Int. J. Robot. Autom. 2014, 3, 234-244. [CrossRef]

15. Suri, A.; Singh, S.K.; Sharma, R.; Sharma, P.; Garg, N.; Upadhyaya, R. Development of Sign Language using Flex Sensors. In Proceedings of the International Conference on Smart Electronics and Communication, ICOSEC 2020, Trichy, India, 10-12 September 2020; pp. 102-106.

16. Sbernini, L.; Pallotti, A.; Saggio, G. Evaluation of a Stretch Sensor for Its Inedited Application in Tracking Hand Finger Movements. In Proceedings of the 2016 IEEE International Symposium on Medical Measurements and Applications (MeMeA), Benevento, Italy, 15-18 May 2016.

17. Manoharan, S.; Ragul, R.; Ramanathan, S.K.S.; Vijay, M.; Palanivel, R. Gesture control of home appliances using flex sensor. Ann. Rom. Soc. Cell Biol. 2021, 25, 4442-4448.

18. Saggio, G.; Lagati, A.; Oreng, G. Wireless Sensory Glove System developed for advanced Human Computer Interface. Int. J. Inf. Sci. 2012, 2, 54-59. [CrossRef]

19. Thanakodi, S.; Azhar, S.M.H.; Miskon, A. Prosthetic hand controlled by wireless flex sensor on EOD robot. IFMBE Proc. 2016, 56, 163-167. [CrossRef]

20. Jabin, J.; Adnan, M.E.; Mahmud, S.S.; Chowdhury, A.M.; Islam, M.R. Low cost 3D printed prosthetic for congenital amputation using flex sensor. In Proceedings of the 2019 5th International Conference on Advances in Electrical Engineering, ICAEE 2019, Dhaka, Bangladesh, 26-28 September 2019; pp. 821-825.

21. Resta, P.; Presti, D.L.; Schena, E.; Massaroni, C.; Formica, D.; Kim, T.; Shin, D. A wearable system for knee flexion/extension monitoring: Design and assessment. In Proceedings of the 2020 IEEE International Workshop on Metrology for Industry 4.0 \& IoT, Roma, Italy, 3-5 June 2020; pp. 273-277.

22. Cochrane, C.; Koncar, V.; Lewandowski, M.; Dufour, C. Design and Development of a Flexible Strain Sensor. Sensors 2007, 7, 473-492. [CrossRef]

23. Linder, A.; Kircher, A.; Vadeby, A. Intelligent Transport Systems (ITS) in Passenger Cars and Methods for Assessment of Traffic Safety Impact; Statens Väg- och Transportforskningsinstitut: Linköping, Sweden, 2007.

24. Salman, F.; Cui, Y.; Imran, Z.; Liu, F.; Wang, L.; Wu, W. A Wireless-controlled 3D printed Robotic Hand Motion System with Flex Force Sensors. Sens. Actuators A Phys. 2020, 309, 112004. [CrossRef]

25. Trung, N.T.; Son, T.H. Using flex sensor to get the angles from master hand to control slave hand. In Proceedings of the ICMSCE 2018: 2018 2nd International Conference on Mechatronics Systems and Control Engineering, Amsterdam, The Netherlands, 21-23 February 2018; pp. 1-4.

26. Rusydi, M.I.; Opera, M.I.; Rusydi, A.; Sasaki, M. Combination of flex sensor and electromyography for hybrid control robot. Telkomnika Telecommun. Comput. Electron. Control 2018, 16, 2275-2286. [CrossRef]

27. Elgeneidy, K.; Lohse, N.; Jackson, M. Bending angle prediction and control of soft pneumatic actuators with embedded flex sensors-A data-driven approach. Mechatronics 2018, 50, 234-247. [CrossRef]

28. Gerboni, G.; Diodato, A.; Ciuti, G.; Cianchetti, M.; Menciassi, A. Feedback Control of Soft Robot Actuators via Commercial Flex Bend Sensors. IEEE/ASME Trans. Mechatron. 2017, 22, 1881-1888. [CrossRef]

29. Gul, J.Z.; Kim, K.H.; Lim, J.H.; Doh, Y.H.; Choi, K.H. FSI modeling of frog inspired soft robot embedded with ALD encapsulated flex sensor for underwater synchronous swim. In Proceedings of the 2017 IEEE 5th International Symposium on Robotics and Intelligent Sensors (IRIS), Ottawa, ON, Canada, 5-7 October 2017; pp. 255-259.

30. Mondal, B.; Ali, S.F.; Mandal, N. An Approach to Design a Wireless Air Flow Transmitter Using Flex Sensor. IEEE Sens. J. 2021, 21, 10498-10505. [CrossRef]

31. Syed, A.; Agasbal, Z.T.H.; Melligeri, T.; Gudur, B. Flex Sensor Based Robotic Arm Controller Using Micro Controller. J. Softw. Eng. Appl. 2012, 5, 364-366. [CrossRef]

32. Yang, Z.; Pang, Y.; Han, X.L.; Yang, Y.; Yang, Y.; Ling, J.; Jian, M.; Zhang, Y.; Ren, T.L. Graphene Textile Strain Sensor with Negative Resistance Variation for Human Motion Detection. ACS Nano 2018, 12, 9134-9141. [CrossRef]

33. Wang, J.; Lin, M.F.; Park, S.; Lee, P.S. Deformable conductors for human-machine interface. Mater. Today 2018, 21, 508-526. [CrossRef]

34. Merilampi, S.; Laine-Ma, T.; Ruuskanen, P. The characterization of electrically conductive silver ink patterns on flexible substrates. Microelectron. Reliab. 2009, 49, 782-790. [CrossRef] 
35. Biddiss, E.; Chau, T. Electroactive polymeric sensors in hand prostheses: Bending response of an ionic polymer metal composite. Med. Eng. Phys. 2006, 28, 568-578. [CrossRef] [PubMed]

36. Zhang, W.; Liu, Q.; Chen, P. Flexible strain sensor based on carbon black/silver nanoparticles composite for humanmotion detection. Materials 2018, 11, 1836. [CrossRef] [PubMed]

37. Serpelloni, M.; Tiboni, M.; Lancini, M.; Pasinetti, S.; Vertuan, A.; Gobbo, M. Preliminary Study of aA Robotic Rehabilitation System Driven by EMG for Hand Mirroring. In Proceedings of the 2016 IEEE International Symposium on Medical Measurements and Applications (MeMeA), Benevento, Italy, 15-18 May 2016.

38. Tiboni, M.; Legnani, G.; Lancini, M.; Serpelloni, M.; Gobbo, M.; Fausti, D. ERRSE: Elbow robotic rehabilitation system with an EMG-based force control. Mech. Mach. Sci. 2018, 49, 892-900. [CrossRef]

39. Amici, C.; Ghidoni, M.; Ceresoli, F.; Gaffurini, P.; Bissolotti, L.; Mor, M.; Fausti, D.; Antonini, M.; Ragni, F.; Tiboni, M. Preliminary Validation of a Device for the Upper and Lower Limb Robotic Rehabilitation. In Proceedings of the 2019 23rd International Conference on Mechatronics Technology (ICMT), Salerno, Italy, 23-26 October 2019; pp. 1-6.

40. Amici, C.; Ragni, F.; Ghidoni, M.; Fausti, D.; Bissolotti, L.; Tiboni, M. Multi-sensor validation approach of an end-effector-based robot for the rehabilitation of the upper and lower limb. Electronics 2020, 9, 1751. [CrossRef]

41. Negrini, S.; Piovanelli, B.; Amici, C.; Cappellini, V.; Bovi, G.; Ferrarin, M.; Zaina, F.; Borboni, A. Trunk motion analysis: A systematic review from a clinical and methodological perspective. Eur. J. Phys. Rehabil. Med. 2016, 52, 583-592. [PubMed]

42. Saggio, G.; Orengo, G. Flex sensor characterization against shape and curvature changes. Sens. Actuators A Phys. 2018, 273, 221-231. [CrossRef]

43. Orengo, G.; Sbernini, L.; di Lorenzo, N.; Lagati, A.; Saggio, G. Curvature Characterization of Flex Sensors for Human Posture Recognition. Univers. J. Biomed. Eng. 2013, 1, 10-15. [CrossRef]

44. Sanca, A.S.; Rocha, J.C.; Eugenio, K.J.S.; Nascimento, L.B.P.; Alsina, P.J. Characterization of Resistive Flex Sensor Applied to Joint Angular Displacement Estimation. In Proceedings of the 15th Latin American Robotics Symposium, 6th Brazilian Robotics Symposium and 9th Workshop on Robotics in Education, LARS/SBR/WRE 2018, João Pessoa, Brazil, 6-10 November 2018; pp. $39-44$.

45. Ash HE, U.A. Proximal Interphalangeal Joint Dimensions for the Design of a Surface Replacement Prosthesis. Proc. Inst. Mech. Eng. Part H J. Eng. Med. 1996, 210, 95-108. [CrossRef] [PubMed]

46. Tiboni, M.; Aggogeri, F.; Pellegrini, N.; Perani, C.A. Smart modular architecture for supervision and monitoring of a 4.0 production plant. Int. J. Autom. Technol. 2019, 13, 310-318. [CrossRef]

47. Tiboni, M.; Incerti, G.; Remino, C.; Lancini, M. Comparison of signal processing techniques for condition monitoring based on artificial neural networks. Appl. Cond. Monit. 2019, 15, 179-188. [CrossRef]

48. Tiboni, M.; Remino, C. Condition Monitoring of a Mechanical Indexing System with Artificial Neural Networks. In Proceedings of the First World Congress on Condition Monitoring 2017 exceeds expectations, London, UK, 13-16 June 2017.

49. Oess, N.P.; Wanek, J.; van Hedel, H.J. Enhancement of Bend Sensor Properties as Applied in A Glove for Use in Neurorehabilitation Settings. In Proceedings of the 2010 Annual International Conference of the IEEE Engineering in Medicine and Biology, Buenos Aires, Argentina, 31 August-4 September 2010; pp. 5903-5906.

50. Yao, C.; Hong, C.; Su, D.; Zhang, Y.; Yin, Z. Design and verification of a wireless sensing system for monitoring large-range ground movement. Sens. Actuators A Phys. 2020, 303, 111745. [CrossRef]

51. Saggio, G. Mechanical model of flex sensors used to sense finger movements. Sens. Actuators A Phys. 2012, 185, 53-58. [CrossRef] 